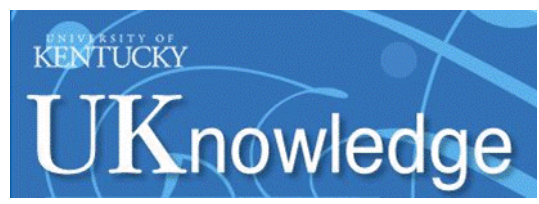

University of Kentucky

UKnowledge

3-10-1995

\title{
Optically Thin Broad-Line Clouds in Active Galactic Nuclei
}

Joseph C. Shields

University of Arizona

Gary J. Ferland

University of Kentucky, gary@uky.edu

Bradley M. Peterson

The Ohio State University

Follow this and additional works at: https://uknowledge.uky.edu/physastron_facpub

Part of the Astrophysics and Astronomy Commons, and the Physics Commons

Right click to open a feedback form in a new tab to let us know how this document benefits you.

\section{Repository Citation}

Shields, Joseph C.; Ferland, Gary J.; and Peterson, Bradley M., "Optically Thin Broad-Line Clouds in Active Galactic Nuclei" (1995). Physics and Astronomy Faculty Publications. 161.

https://uknowledge.uky.edu/physastron_facpub/161

This Article is brought to you for free and open access by the Physics and Astronomy at UKnowledge. It has been accepted for inclusion in Physics and Astronomy Faculty Publications by an authorized administrator of UKnowledge. For more information, please contact UKnowledge@lsv.uky.edu. 


\section{Optically Thin Broad-Line Clouds in Active Galactic Nuclei}

\section{Digital Object Identifier (DOI)}

http://dx.doi.org/10.1086/175378

\section{Notes/Citation Information}

Published in The Astrophysical Journal, v. 441, no. 2, p. 507-520.

(C) 1995. The American Astronomical Society. All rights reserved.

The copyright holder has granted permission for posting the article here. 


\title{
OPTICALLY THIN BROAD-LINE CLOUDS IN ACTIVE GALACTIC NUCLEI
}

\author{
Joseph C. Shields, ${ }^{1,2}$ Gary J. Ferland, ${ }^{3}$ And Bradley M. Peterson ${ }^{4}$ \\ Received 1994 March 28; accepted 1994 September 19
}

\begin{abstract}
The broad-line region (BLR) in Seyfert galaxy nuclei exhibits correlated variations in continuum and emission-line luminosity that are qualitatively consistent with photoionization of ionization-bounded (optically thick) clouds. However, evidence is growing that a nonnegligible fraction of the BLR cloud population is optically thin to the Lyman continuum and fully ionized in hydrogen. We consider the implications of this nebular component for observed line emission and find that inclusion of thin clouds in photoionization calculations can resolve several outstanding puzzles of Seyfert variability, notably the behavior of the C IV $\lambda 1549$ / Ly $\alpha$ ratio as a function of continuum luminosity.

A similar population of thin clouds located along our line of sight can account for observed ultraviolet absorption features and "warm absorber" behavior at X-ray energies. The Baldwin effect for active galaxies, a negative correlation between ultraviolet emission-line equivalent width and continuum luminosity, can also be explained in detail by a decrease in the covering factor of an optically thin component with increasing source luminosity. The luminosity dependence of covering factor may result from outflows of thin clouds that proceed more efficiently in intrinsically brighter sources. The presence of absorption features in AGNs that are blueshifted and attain the highest velocities in broad absorption line features associated with luminous QSOs would be consistent with this interpretation.
\end{abstract}

Subject headings: galaxies: active — galaxies: nuclei - galaxies: Seyfert - quasars: emission lines

\section{INTRODUCTION}

Variability monitoring has provided new insights into the structure of active galactic nuclei (AGNs) while also presenting new challenges to the physical understanding of these sources. The emission-line gas in AGN broad-line regions (BLRs) can be understood in general terms as photoionized plasma, and luminosity variations in the emission lines and observable continuum are indicative of correlated variations in the ionizing radiation field (e.g., Peterson 1993). Understanding of the detailed aspects of emission-line variability remains primitive, however.

The direct correlation in individual Seyfert nuclei between the luminosity in the continuum and in emission lines, including recombination features, implies that a large part of the emitting gas resides in clouds that contain an ionization front, such that the emission measure of nebular matter within a cloud scales directly with the incident ionizing continuum. The previous generation of BLR photoionization models generally assumed that a single population of clouds was responsible for all emission properties, necessitating large cloud optical depths in the Lyman continuum in order to produce strong emission from both high- and low-ionization species (e.g., $\mathrm{C}$ IV and $\mathrm{Fe}$ II; Ferland \& Persson 1989; but see Collin-Souffrin et al. 1986 for a two-component alternative). However, time-delay analysis of AGN variability data has since demonstrated that responsivity in different lines is radially stratified, such that low-ionization line response originates from characteristically larger distances

\footnotetext{
${ }^{1}$ Present address: Steward Observatory, University of Arizona, Tucson, AZ 85721. Also Department of Astronomy, The Ohio State University; and Department of Physics and Astronomy, University of Kentucky.

${ }^{2}$ Hubble Fellow.

${ }^{3}$ Department of Physics \& Astronomy, University of Kentucky, Lexington, KY 40506.

${ }^{4}$ Department of Astronomy, The Ohio State University, Columbus, $\mathrm{OH}$ 43210.
}

from the central source than response from high-ionization species. Large column densities are consequently not a requirement for BLR clouds in general, and the possibility thus exists that a substantial part of the BLR is optically thin to Lymancontinuum radiation.

Several observational results suggest, with varying degrees of confidence, the presence within the BLR of gas that is optically thin to the Lyman continuum. Wamsteker \& Colina (1986) noted that the luminosity of C IV emission from Fairall 9 appears to saturate when the continuum is in a high phase (the "Wamsteker-Colina effect") and argued that this pattern results from the transition of BLR clouds to a fully ionized state. $^{5}$ Emission in C IV is now known to respond to the continuum level with a significant delay in Fairall 9 ( $\sim 200$ days; Clavel, Wamsteker, \& Glass 1989). It is thus conceivable that the Wamsteker-Colina effect could be due in part to the temporal offset between line and continuum implicit in a single epoch of observation. Emission in C IV is moreover sensitive to details of the incident continuum spectral energy distribution (SED), as well as density and other physical properties of the BLR clouds, which could evolve in response to elevated radiation densities. In the case of Mrk 590, however, apparent saturation of emission in the high-velocity wings of Balmer lines has been observed (Ferland, Korista, \& Peterson 1990).

Other evidence for optically thin material in the BLR comes from analyses comparing line profiles of emission lines that trace thick and thin components. Morris \& Ward (1989) compared emission-line profiles for $\mathrm{H} \alpha$ and $\mathrm{O}$ I $\lambda 8446$ in an effort to distinguish $\mathrm{H} \alpha$ contributions from optically thin material that does not generate significant $\mathrm{O}_{\mathrm{I}}$ emission. They found measurable profile differences in only a fraction of their sample objects, which they regarded as evidence that optically thin

\footnotetext{
5 In the present discussion, the terminology "fully ionized" is used with reference to hydrogen only.
} 
material is usually not a significant contributor to BLR emission. Morris \& Ward noted, however, that optically thin gas could nonetheless contribute substantially to $\mathrm{H} \alpha$ if thick and thin components trace the BLR velocity distribution with equal weighting. Modest contributions to $\mathrm{H} \alpha$ inferred from the profile comparison could moreover implicate relatively large contributions to $\operatorname{Ly} \alpha$ and other lines that form primarily in highly ionized cloud components, rather than the high column density medium that is probably responsible for most emission in the Balmer lines and from low-ionization species. This point was emphasized by Zheng (1992), who argued on the basis of profile comparisons between $\mathrm{H} \alpha$ and $\mathrm{Ly} \alpha$ that an optically thin region could contribute as much as $10 \%$ of the total Balmer lines and $50 \%$ of the total Ly $\alpha$ in a few objects.

The case for optically thin clouds within the BLR has been strengthened recently by Sparke (1993), who analyzed ultraviolet monitoring data for NGC 5548. Her results show that the autocorrelation of line flux is sometimes more narrowly peaked than that of continuum flux, and also that the crosscorrelation of line and continuum flux appears steeper on the negative time lag side (of line relative to continuum). Both findings are most readily interpreted as evidence that line emission from the inner BLR actually decreases as the continuum brightens. Fully ionized clouds can show a negative correlation between incident flux and emitted radiation in specific lines (§ 2). If the BLR clouds are illuminated by a central source and have column densities independent of their distance from that source, such clouds are more likely to be fully ionized if situated at small nuclear distances where the flux of ionizing radiation is high. The cross-correlation results for NGC 5548 can thus be readily understood as a consequence of optically thin clouds within the inner BLR. Alternative physical mechanisms that can account for this behavior are not obvious.

Optically thin circumnuclear clouds are also clearly identifiable in absorption. Roughly $10 \%$ of QSOs exhibit broad absorption lines (BALs) produced by near-nucleus material along the line of sight that contains a low neutral fraction (Foltz et al. 1990; Weymann et al. 1991). Less dramatic absorption by clouds that are probably optically thin is also common in Seyfert nuclei (e.g., Ulrich 1988). Low-luminosity AGNs often show the signatures of soft-X-ray-absorbing gas (column density $\sim 10^{21}-10^{23} \mathrm{~cm}^{-2}$ ) along the line of sight that may constitute a portion of the BLR (e.g., Reichert et al. 1985). Some Seyfert nuclei exhibit variable X-ray emission that is inversely correlated with the apparent column density of absorbing material; such behavior is most readily interpreted as the hallmark of a "warm absorber" that varies in soft X-ray opacity as its ionization state fluctuates in response to continuum intensity (Halpern 1984). Circumnuclear material that is identifiable as a warm absorber is unambiguously optically thin to the Lyman continuum. Whether a population of clouds similar to those seen in absorption will also be important sources of line emission will depend on the number of such clouds residing outside of our line of sight. Plasma that is sufficiently ionized to act as a warm absorber may also be an inefficient radiator of optical and ultraviolet lines.

Given the increasing evidence that optically thin nebular components may constitute an important part of the BLR in variable Seyfert galaxies, we have performed new photoionization calculations that demonstrate some of the properties of such material $(\S 2)$. We find that inclusion of an optically thin component in BLR representations can help explain several puzzling aspects of emission-line variability in Seyfert nuclei
(§ 3). In particular, a mix of thick and thin clouds can account for the nonlinear response of emission lines to continuum changes, including the negative correlation between the $\mathrm{C}$ IV $\lambda 1549 /$ Ly $\alpha$ ratio and continuum brightness observed in some sources. This correlation has proven difficult or impossible to account for with a population of thick clouds alone. When seen along the line of sight to the continuum source, a similar population can produce the signatures of a cold or warm absorber commonly seen in soft X-ray data; the physical characteristics of this medium can be constrained in detail by its ultraviolet absorption spectrum. A luminosity-dependent contribution of line emission from thin clouds in the BLR may provide a simple interpretation of the Baldwin effect, a negative correlation between emission-line equivalent width and luminosity observed in AGN ensembles (§ 4).

\section{PROPERTIES OF FULLY IONIZED CLOUDS}

\subsection{Ultraviolet Emission Lines}

In this section we provide an illustrative example of emission-line behavior expected for BLR clouds that become fully ionized. Photoionization calculations relevant to BLR emission nebulae were carried out with the code Cloudy, version 84.09 (Ferland 1993). Clouds were assumed to have a constant hydrogen density $n$ of $10^{11} \mathrm{~cm}^{-3}$, which is consistent with current estimates for the inner BLR (Ferland et al. 1992). Solar abundances were assumed (Grevesse \& Anders 1989) and are consistent with available constraints for Seyfert 1 nuclei (Hamann \& Ferland 1993). The ionizing spectral energy distribution is the average AGN continuum described by Mathews \& Ferland (1987), modified by a break to a power law with spectral index $\alpha=2.5\left(f_{v} \propto v^{\alpha}\right)$ longward of $1 \mu \mathrm{m}$. This continuum has $\alpha=-1.0$ at optical wavelengths, and $\alpha=-0.5$ for $4400 \AA>\lambda>520 \AA$, steepening to $\alpha=-1.0$ in the extreme ultraviolet region, with a break to $\alpha=-3$ shortward of $220 \AA$. The X-ray power law is described by $\alpha=-0.7$ between 0.365 and $100 \mathrm{keV}$, with $\alpha=-1.67$ at higher energies. With this prescription, the two-point spectral index connecting $2500 \AA$ and $2 \mathrm{keV}\left(\alpha_{o x}\right)$ is -1.4 .

A cloud becomes fully ionized and thus optically thin to the Lyman continuum at an ionization parameter $U$ (the ratio of ionizing photon to nucleon density) proportional to the cloud's hydrogen column density $N$. Figure 1 displays predicted fluxes for strong emission lines as a function of $U$, emergent from a cloud with $N=10^{23} \mathrm{~cm}^{-2}$; a similar figure can be found in Netzer (1990a). Lyman- $\alpha$ grows approximately linearly in luminosity with increasing $U$ until the cloud becomes fully ionized at $\log U \approx-0.5$. At higher $U$, the volume of recombining gas no longer increases and Ly $\alpha$ luminosity from the cloud remains approximately constant. At $\log U>1$, the emitted Ly $\alpha$ undergoes a decline due to decreasing recombination rates accompanying a significant increase in nebular temperature (i.e., average $\log T_{e}=5.6,5.7,5.9,6.4$ for $\log U=0.5,1.0,1.5$, 2.0).

Emission in the other lines plotted in Figure 1 varies with $U$ in a manner depending on where the line forms relative to the hydrogen ionization front. He II $\lambda 1640$ continues to increase in luminosity after the cloud is fully ionized, reflecting the presence of a $\mathrm{He}^{+} / \mathrm{He}^{++}$ionization front interior to the ionized hydrogen zone. He II emission reaches a maximum at $\log U \approx 0.5$ when the helium ionization front reaches the back of the cloud, and the line decreases at higher $U$ in resemblance to Ly $\alpha$. Emission in moderately high ionization lines 


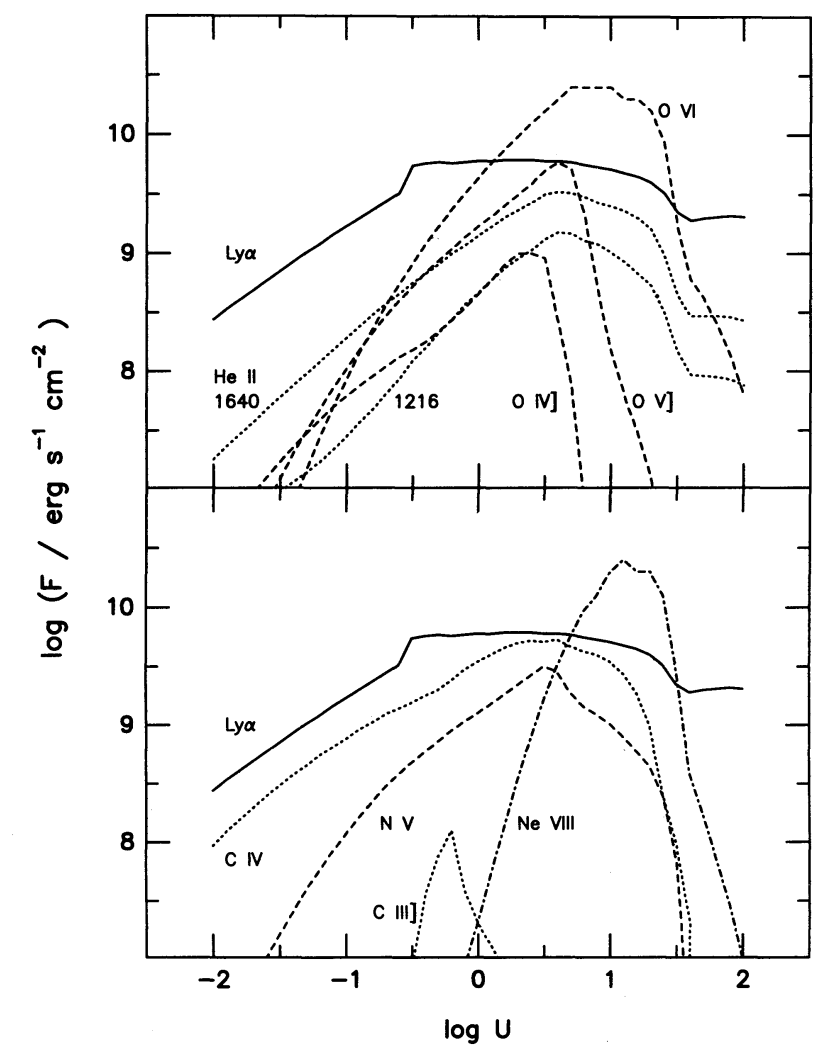

FIG. 1.-Emission-line fluxes as a function of $U$, for clouds with $n=10^{11}$ $\mathrm{cm}^{-3}$ and $N=10^{23} \mathrm{~cm}^{-2}$, irradiated by the standard AGN continuum. The indicated features correspond to Ne viII $\lambda 774, \mathrm{O}$ vi $\lambda 1035$, Ly $\alpha \lambda 1216$, $\mathrm{He}$ II $\lambda 1216, \mathrm{O}$ v] $\lambda 1218, \mathrm{~N}$ v $\lambda 1240, \mathrm{O}$ Iv] $\lambda 1400, \mathrm{C}$ IV $\lambda 1549, \mathrm{He}$ II $\lambda 1640$, and C III] $\lambda 1909$.

such as $\mathrm{C}$ Iv $\lambda 1549, \mathrm{~N} v \lambda 1240$, and $\mathrm{O} v \mathrm{v}] \lambda 1218$ shows a general similarity to $\mathrm{He}$ II in responding to changes in $U$, although each of these heavy-element lines decreases much more abruptly at high $U$ due to a transition of these elements to characteristically higher ionization. The transition between dominant ionization states is demonstrated more explicitly in the progression of $U$ represented by maximum emission in $\mathrm{O}$ IV] $\lambda 1400, O \mathrm{v}] \lambda 1218$, and $\mathrm{O}$ vi $\lambda 1035$. The great strength of $\mathrm{O}$ vI and $\mathrm{Ne}$ viII $\lambda 774$ emission at high $U$ provides an important signature of high-ionization nebular components. A possible role for optically thin clouds in producing AGN emission in high-ionization lines has been noted in previous studies (e.g., Netzer 1976; Davidson 1977).

A measure of the significance of predicted emission-line flux relative to observed lines is provided by comparison of predicted and observed equivalent widths. The equivalent width of $\operatorname{Ly} \alpha, \mathrm{EW}(\mathrm{Ly} \alpha)$, is plotted as a function of $U$ in Figure $2 a$, in the limit of $100 \%$ coverage; more general estimations of $\mathrm{EW}(\mathrm{Ly} \alpha)$ require a scaling of these predictions by the fractional covering factor $f_{c}$. Measured $\operatorname{EW}(\operatorname{Ly} \alpha)$ for variable Seyfert nuclei is typically of order $100 \AA$. The figure shows that $\mathrm{EW}(\mathrm{Ly} \alpha)$ decreases gradually with increasing $U$ until the cloud becomes fully ionized, beyond which $\mathrm{EW}(\mathrm{Ly} \alpha)$ decreases much more rapidly, with a logarithmic slope of approximately -1 . In this example, an optically thin cloud needs to be described by $\log U<0.5$ in order for such a nebular component to contribute half of the Ly $\alpha$ emission in a typical Seyfert nucleus. This bound is obviously decreased further if $f_{c}<1$. For comparison, a similar plot of $\mathrm{EW}(\mathrm{Ly} \alpha)$ versus $U$ is shown in Figure

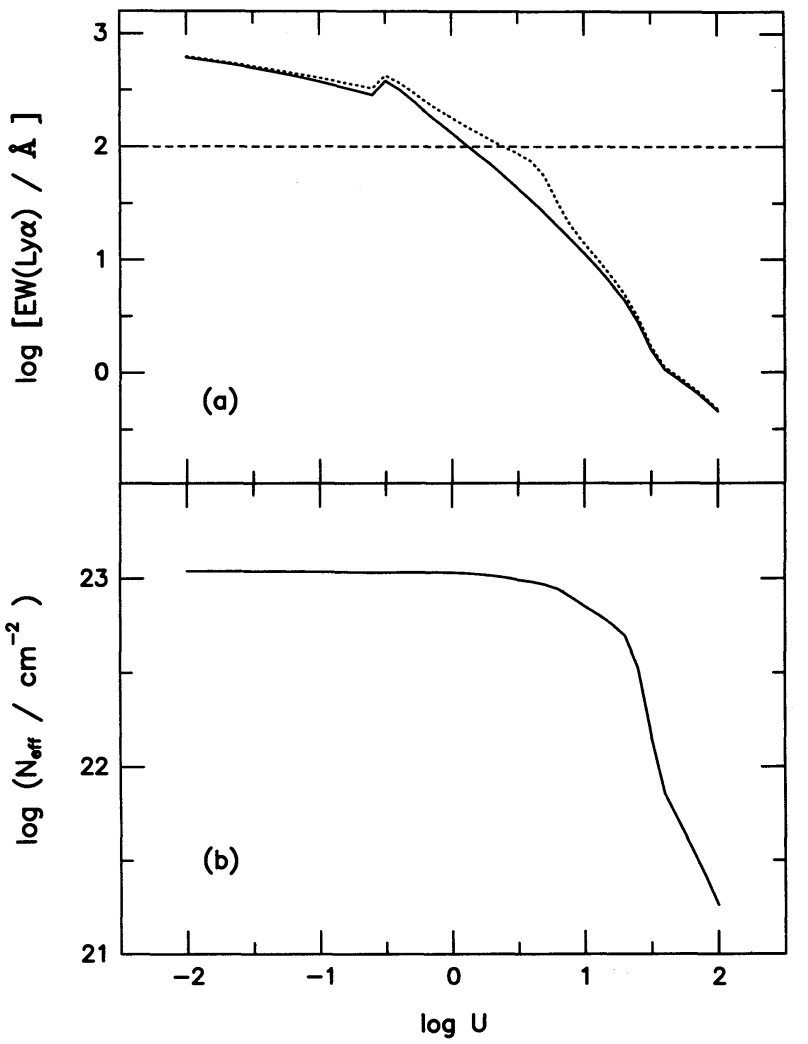

FIG. 2.- (a) Equivalent width of Ly $\alpha$ (solid curve) as a function of $U$ for the calculations shown in Fig. 1, assuming full coverage $\left(f_{c}=1\right)$. The dashed line indicates the typical equivalent width observed in AGNs. The dotted curve represents the equivalent width of blended $\mathrm{Ly} \alpha, \mathrm{He}$ II $\lambda 1216$, and $\mathrm{O}$ v]. The jump at $\log U \approx-0.5$ occurs when the hydrogen ionization front reaches the back of the cloud, leading to an increase in the emission-line escape probability. (b) Apparent column density of the same nebular clouds, inferred from the transmitted flux at $1 \mathrm{keV}$ and assuming a cold absorbing medium.

$3 a$ for the case when $N$ is reduced to $10^{21} \mathrm{~cm}^{-2}$. The cloud is fully ionized throughout the plotted range of $U$, and $\mathrm{EW}(\operatorname{Ly} \alpha)$ decreases steadily with a logarithmic slope near -1 . For clouds that are fully ionized (matter-bounded), Figures $2 a$ and $3 a$ illustrate that $\mathrm{EW}(\mathrm{Ly} \alpha)$ predicted at a given $U$ scales with emission measure and hence with $N$.

Given the fundamental role of $\mathrm{Ly} \alpha$ emission in gaseous nebulae, it is often convenient to gauge nebular behavior in terms of $\mathrm{EW}(\operatorname{Ly} \alpha)$ or line intensity normalized to Ly $\alpha$ strength. However, emission in this feature measured in AGNs is likely to include blended contributions from $\mathrm{He}$ II $\lambda 1216$ and $\mathrm{O} v$ ] $\lambda 1218$ (Ferland et al. 1992). Previous BLR photoionization calculations have generally ignored these blended components when comparing theoretical results with observed line ratios. For the "old" standard BLR cloud described by $\log U \approx-2$ and $n \approx 10^{10} \mathrm{~cm}^{-3}$ (e.g., Davidson \& Netzer 1979), this is a reasonable procedure, since the contributions of $\mathrm{He}$ II and $O \mathrm{v}]$ are expected to be negligible. However, for higher $n$ and $U$, and especially for optically thin conditions, these features can be strong relative to predicted Ly $\alpha$. For the calculations presented in Figure 1, He II $\lambda 1216$ and $\mathrm{O}$ v] $\lambda 1218$ reach maxima relative to $L y \alpha$ of $\sim 0.2$ and $\sim 1$, respectively, at $\log$ $U=0.5$, implying a difference between the predicted Ly $\alpha$ and observationally relevant blend of more than a factor of 2 . The 


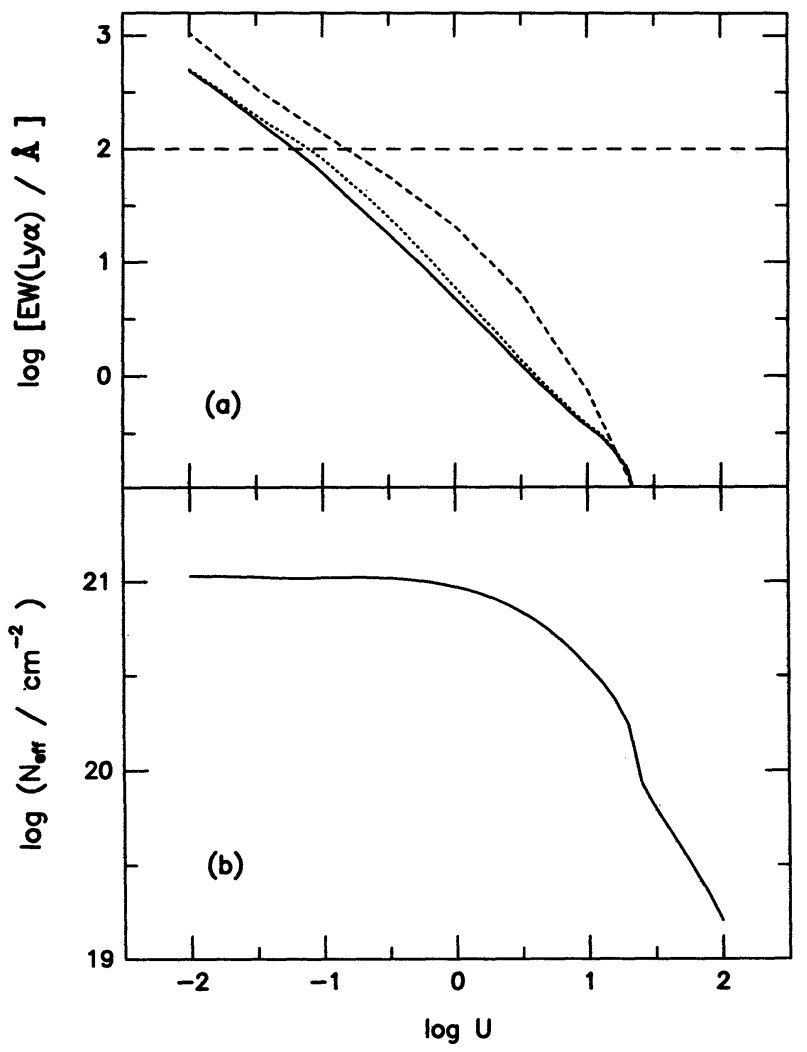

Fig. 3.-Equivalent width of $\operatorname{Ly} \alpha$ (solid curve) as a function of $U$ when $N$ is reduced to $10^{21} \mathrm{~cm}^{-2}$. The dotted curve represents the equivalent width of the Ly $\alpha$ blend. The dashed curve shows the equivalent width of the Ly $\alpha$ blend when the cloud is modified by turbulence represented by $b_{\text {turb }}=5000 \mathrm{~km} \mathrm{~s}^{-1}$. (b) Apparent column density of the same nebular clouds, inferred from the transmitted flux at $1 \mathrm{keV}$ and assuming a cold absorbing medium.

variation of apparent $\mathrm{EW}(\mathrm{Ly} \alpha)$ versus $U$, inclusive of the blended lines, is shown by the dotted line in Figure $2 a$. The equivalent width of emission in the apparent $\operatorname{Ly} \alpha$ feature would thus remain consistent with observed values at $\log U$ as large as 0.5 . The influence of a blended component at $1218 \AA$ is also of potential significance in the interpretation of relative velocity shifts between Ly $\alpha$ and other AGN emission lines (e.g., Tytler \& Fan 1992). The predicted equivalent width of the Ly $\alpha$ blend is shown in Figure $3 a$ for the cloud with $N=10^{21} \mathrm{~cm}^{-2}$; contamination by $\mathrm{He}$ II and $\mathrm{O} \mathrm{v}$ ] is again nonnegligible.

In the preceding discussion, the line fluxes emitted by a thin cloud have been calculated assuming a thermal line width. In an alternative picture, the emitting plasma may be comprised of filaments or other structures described internally by thermal gas motions, but in the aggregate by a much broader velocity distribution resulting from turbulence or other bulk motion. If the mean free path for scattering line photons exceeds the size scale of a single filament, the line width is described by the aggregate velocity width, rather than simply a thermal width. In the case of BLR clouds, the difference in the two widths may be up to about three orders of magnitude if the bulk motion of the thin substructures corresponds to the observed line width.

The consequences of adding velocity structure to the thin clouds are shown in Figure 4 for line emission from a cloud with $n=10^{11} \mathrm{~cm}^{-3}, N=10^{21} \mathrm{~cm}^{-2}$, irradiated by the standard AGN continuum. In this plot, $\log U$ is fixed at -1 , and a microturbulent velocity $b_{\text {turb }}$ (added in quadrature to the

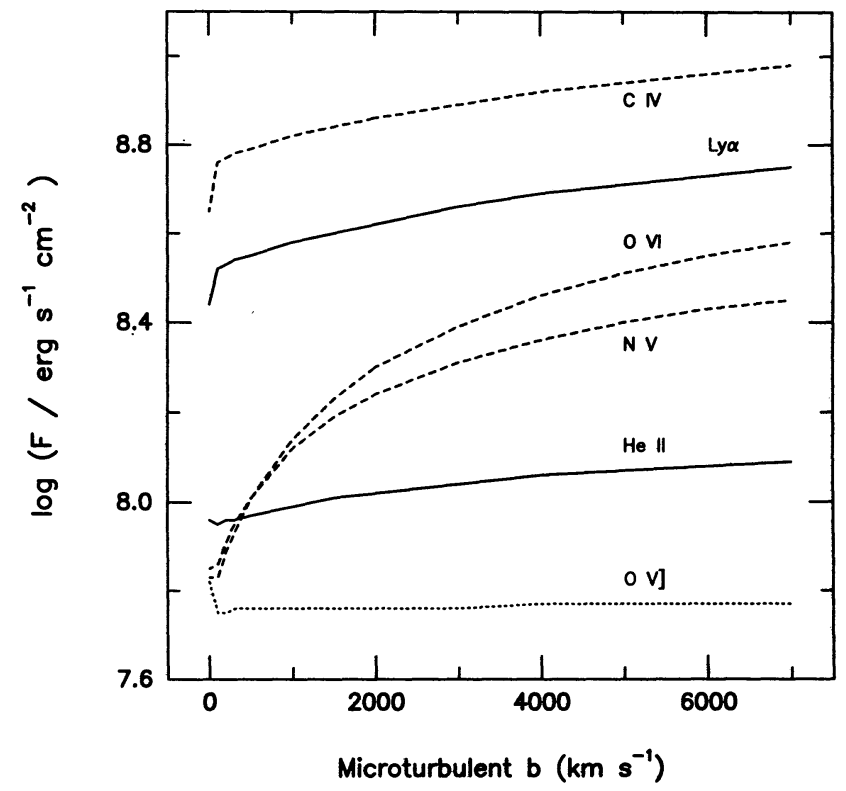

FIG. 4. - Line flux as a function of $b_{\text {turb }}$ produced by a cloud with $n=10^{11}$ $\mathrm{cm}^{-3}$ and $N=10^{21} \mathrm{~cm}^{-2}$, subject to $\log U=-1$ with the standard AGN continuum. The $\mathrm{He}$ il line is $\lambda 1640$.

thermal $b \equiv[2 k T / m]^{0.5}$ ) is varied. (Assuming a Gaussian profile, $\sigma \approx 0.7 b$ and full width at half-maximum $\approx 1.7 b$.) The permitted lines undergo a significant increase in emitted flux as $b_{\text {turb }}$ grows. Lines with high optical depth that are partially thermalized (e.g., C Iv, Ly $\alpha$ ) undergo an initial jump in emitted flux as the line width is increased from the thermal value. The growth in line flux accompanying further increases in $b_{\text {turb }}$ and seen in other lines stems primarily from increasing continuum fluorescence in the optically thick lines as the line width grows. Bulk motion within thin clouds thus may significantly increase the equivalent width of permitted lines generated in such a medium. The equivalent width of the Ly $\alpha$ blend as a function of $U$ produced by a cloud with $n=10^{11} \mathrm{~cm}^{-3}, N=10^{21}$ $\mathrm{cm}^{-2}$, and $b_{\text {turb }}=5000 \mathrm{~km} \mathrm{~s}^{-1}$ is shown in comparison with the $b_{\text {turb }}=0$ case in Figure $3 a$.

While the present calculations illustrate the consequences of turbulent line widths in general terms, additional complications may affect predictions appropriate for detailed comparisons with observations. In particular, added velocity width can cause the profiles of adjacent lines (e.g., Ly $\alpha$ and $\mathrm{N}$ v) to overlap, leading to modifications of line transfer that are not included in the current treatment. The consequences for the emergent spectrum will depend on the detailed geometry and velocity structure of the line-emitting gas. In cases in which the radiation is important in determining the dynamical properties of circumnuclear plasma, the consequences of line overlap may be especially profound (e.g., Korista et al. 1993 and references therein).

Thin, fully ionized clouds within the BLR will also have the important property of being essentially isotropic emitters of line radiation. Within the inner BLR, a cloud that is optically thick to the ionizing continuum is expected to radiate line emission highly anisotropically, with most of the line luminosity emergent from the illuminated face of the cloud (Ferland et al. 1992). The asymmetry arises from the large line optical depths, potentially aided by continuum opacity impeding escape from the back of the cloud. This prediction takes on 
particular significance in conjunction with the observational finding that Ly $\alpha$ and possibly other ultraviolet lines are highly symmetric in profile (Laor et al. 1994 and references therein). If the BLR clouds are thick, the observed line luminosity will be dominated by clouds on the far side of the central engine, and the observed line symmetry precludes a purely radial flow for the clouds. If thin clouds are an important constituent of the BLR, however, the observed line emission will trace the full velocity field, and flows that are spherically or axially symmetric or turbulent would be consistent with the observed profiles. When subject to very high $U$, fully ionized clouds enter a hot phase that is an inefficient emitter of line radiation (Fig. 1). This medium may still play an important role in generating symmetric line profiles if its column density is sufficient to be optically thick to electron scattering $\left(N \gtrsim 10^{24} \mathrm{~cm}^{-2}\right)$; the observed line width may then arise primarily from broadening in the scattering process rather than from bulk motion (e.g., Weymann 1970; Emmering, Blandford, \& Shlosman 1992).

If thin clouds contribute nonnegligible line emission in a significant fraction of AGNs, the long-standing problem of explaining the low ratio of $\mathrm{Ly} \alpha / \mathrm{H} \beta$ will be exacerbated, a point emphasized by Voit, Shull, \& Begelman (1987). Theoretical attempts to explain the observed ratio usually invoke substantial emission from very thick clouds (e.g., Kwan \& Krolik 1981). If substantial $\mathrm{Ly} \alpha$ is generated in thin clouds producing a large $\mathrm{Ly} \alpha / \mathrm{H} \beta$ ratio, an additional component of presumably very thick clouds must then generate emission described by a $\mathrm{Ly} \alpha / \mathrm{H} \beta$ ratio that is smaller than even the low composite ratio. Accurate calculation of $\mathrm{Ly} \alpha / \mathrm{H} \beta$ predictions for such thick clouds may require a more detailed treatment of radiative transfer than is provided by the escape probability formalism employed in the majority of existing BLR photoionization studies, however (e.g., see discussions in Avrett \& Loeser 1988 and Netzer 1990b), and may be sensitive to detailed cloud parameters and geometry. In the absence of a robust understanding of the hydrogen spectrum for very thick clouds, the extent to which the $\mathrm{Ly} \alpha / \mathrm{H} \beta$ ratio limits the presence of thin clouds in the BLR remains ambiguous.

\subsection{Ultraviolet Line Absorption}

Broad-line region clouds falling along the line of sight to the central continuum source are expected to produce resonance absorption lines, since the source function of BLR clouds is evidently significantly less than that of the agent producing the continuum (Voit et al. 1987). Significant line emission can still be expected from an ensemble of such clouds within the BLR so long as the coverage in velocity space along the line of sight to the continuum source is not large and coverage in positional space outside the line of sight is nonnegligible. If line emission is dominated by continuum fluorescence, however, scattering by clouds along the line of sight will introduce net absorption for a spherically symmetric system except in the limit of $f_{c}=1$. Absorption of line emission from background BLR components will depend on the projected coverage and the relative source functions of the background and foreground clouds. Prediction of the degree of line absorption accompanying a population of thin BLR clouds will thus depend on specific assumptions of geometry and cloud properties.

Absorption line strengths will obviously reflect the column density of absorbing species in the intervening medium. For the cloud with $n=10^{11} \mathrm{~cm}^{-3}$ and $N=10^{21} \mathrm{~cm}^{-2}$ discussed previously, the abundance fraction represented by species responsible for common UV absorption features is shown as a function of $U$ in Figure 5. (Note that these results are relatively insensitive to choice of $n$ ). The plotted values can be converted directly to column densities by scaling by $N$ and the corresponding total abundance, where

$$
\mathrm{C}: \mathrm{N}: \mathrm{O}: \mathrm{Mg}: \mathrm{Si}=-3.44:-3.95:-3.07:-4.42:-4.45
$$

are logarithmic abundances by number relative to hydrogen. Relating observed equivalent widths to ionic column densities is complicated by uncertainty in the intrinsic velocity distribution of absorbers. Minimum column densities can be derived from absorption-line measurements by assuming that the observed absorption occurs on the linear part of the curve of growth (Bromage et al. 1985; Voit et al. 1987; Shull \& Sachs 1993).

Study of absorption features in Seyfert nuclei with good spectral and temporal resolution can yield a number of detailed constraints on the clouds. High-resolution spectroscopy can reveal the presence of structure within a broad absorption profile indicative of high- $N$ absorbing components described by small $b$ values. Measurement of weak absorption from excited states can be used to place constraints on $n$, as discussed by Bromage et al. (1985), who used this method to estimate $n \gtrsim 10^{8.5} \mathrm{~cm}^{-3}$ for absorbing material in NGC 4151 . Variations in ionic column densities in response to changing continuum luminosities provide another means of constraining $n$, using recombination timescale arguments. Estimates derived to date from this method have yielded $n \gtrsim 10^{5} \mathrm{~cm}^{-3}$ for NGC 3516 (Voit et al. 1987), NGC 4151 (Anderson 1974; Bromage et al. 1985), and NGC 5548 (Shull \& Sachs 1993). Changes in inferred ionic column densities in response to variations in continuum luminosity can also be used to constrain $U$, particularly if several species spanning a range of ionization are observed (cf. Fig. 5; see also Smith \& Penston 1988).

Combined estimates of $n, U$, and the ionizing luminosity of the central engine provide a means for determining the radial location of the absorber; lower limits on $n$ translate into upper bounds on distance from the continuum source. While the depth of absorption features has been used as an argument that absorbing systems must reside outside the BLR in some cases (e.g., Mathur et al. 1994), the absorbing clouds could in fact

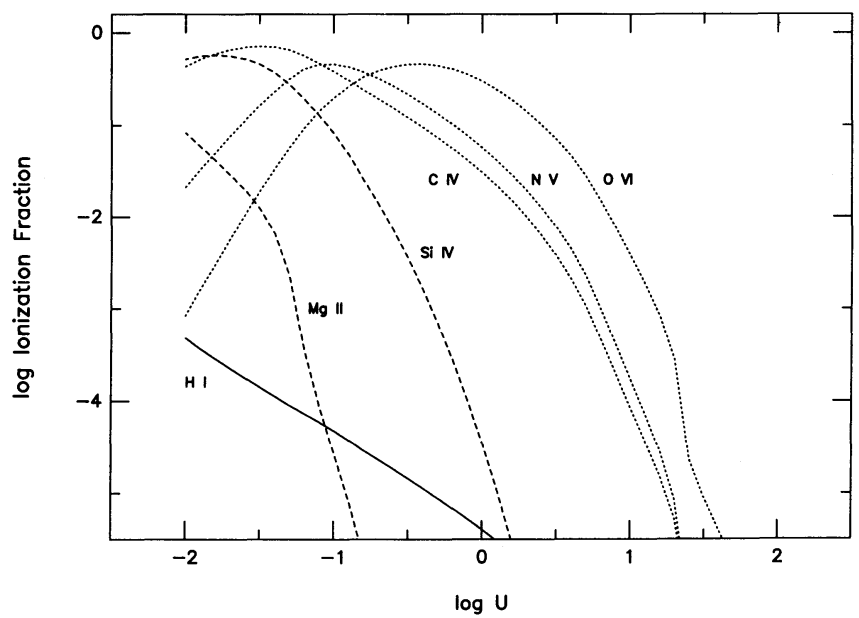

FIG. 5.-Ionization fraction of indicated elements as a function of $U$ for the cloud with $N=10^{21} \mathrm{~cm}^{-3}$. 
reside within the BLR if the BLR emission is emitted highly anisotropically towards the central engine by thick clouds. In this situation, the emission may originate primarily from thick clouds on the far side of the BLR, while absorption results from thin clouds on the near side of the BLR (Shields 1994).

\section{3. $X$-Ray Absorption}

Thin BLR clouds along the line of sight to the continuum source will also have signatures of X-ray absorption. For a plasma in which the heavy elements contributing opacity are only weakly ionized, the absorption will correspond to that from a "cold" absorber. When subject to high $U$, however, increased stripping of the heavy elements will lead to a reduction in opacity at soft energies, corresponding to the behavior of a "warm absorber" (Halpern 1984; Krolik \& Kallman 1984). The detailed physical state of a warm absorber can be constrained from features in high-resolution X-ray spectra (e.g., Netzer 1993). Inclusion of warm absorbers in spectral modeling of existing data can produce good agreement for some AGNs, although the uniqueness of such an interpretation tends to be uncertain, and other scenarios (e.g., partial covering, non-power-law intrinsic spectra) can be difficult to exclude (e.g., Fiore et al. 1993; Kolman et al. 1993; but see Turner et al. 1993). The ability to discern absorption edges in AGN spectra has improved with the advent of $A S C A$, and detailed study will be possible with future data from $A X A F$.

In existing X-ray data, stronger evidence for the presence of a warm absorber may be found in temporal monitoring of variable AGNs that shows an inverse correlation between soft $\mathrm{X}$-ray opacity and continuum state most readily interpreted in terms of changes in ionization state of an intervening medium (Halpern 1984). In studies to date, such behavior is often expressed in terms of a negative correlation between X-ray luminosity and the foreground column density derived assuming a cold absorber.

For comparison with the empirical X-ray results, we consequently define an effective column density $N_{\text {eff }}$, which is calculated for the transmitted continuum at $1 \mathrm{keV}$ assuming absorption by neutral foreground gas. Figure $2 b$ shows $N_{\text {eff }}$ as a function of $U$ for the cloud with $n=10^{11} \mathrm{~cm}^{-3}, N=10^{23}$ $\mathrm{cm}^{-2}$, irradiated by the standard AGN continuum. The diagram shows $N_{\text {eff }}$ decreasing slowly from $N$ as $U$ initially increases. This behavior continues after the cloud becomes optically thin to the Lyman continuum ( $\log U>-0.5)$, but steepens at $\log U \gtrsim 0.5$. For solar abundances, opacity at $\sim 1$ $\mathrm{keV}$ for a plasma with significant ionization is dominated by $\mathrm{K}$ - and L-shell photoionization of oxygen. Significant changes in $N_{\text {eff }}$ can thus occur when $U$ is sufficiently large to produce substantial change in the proportions of partially and fully ionized $\mathrm{O}$.

Detected examples of warm absorbers generally exhibit changes of a factor of 2 or more in $N_{\text {eff }}$ for comparable changes in the medium energy or hard X-ray continuum flux (e.g., Yaqoob, Warwick, \& Pounds 1989; Pan, Stewart, \& Pounds 1990; Nandra, Pounds, \& Stewart 1990; Nandra et al. 1991). If we assume that $U$ scales with the hard X-ray continuum flux (implying a homologous SED throughout the continuum fluctuation), we can compare the observed behavior with Figure $2 b$. For the plotted example, the empirical trend can be reproduced when $\log U \gtrsim 1$.

A comparison of Figures 1 and $2 b$ shows that the flux emitted in most of the strong lines is declining rapidly with increasing $U$ in the warm absorber regime. Exceptions to this pattern are the lines of $\mathrm{O}$ VI and $\mathrm{Ne}$ VIII, for $\log U \lesssim 1.5$, and Ly $\alpha$. The Ne viII line in particular traces media that is likely to act as a warm absorber; emission in $\mathrm{O}$ VI and Ly $\alpha$ can be strong at lower $U$ values where the gas would appear as essentially a cold absorber. Reports have recently emerged of AGNs in which Ne VIII $\lambda 774$ has been detected in broad emission (Hamann et al. 1994) and also absorption (Hamann et al. 1995). These findings bolster the interpretation of warm absorbers as a high-ionization extension of the BLR.

A more general identification of warm absorber media with clouds generating BLR emission will not result if $N$ is changed. The behavior of $N_{\text {eff }}$ as a function of $U$ for a cloud with a smaller $N$ of $10^{21} \mathrm{~cm}^{-2}$ is shown for comparison in Figure $3 b$. Warm-absorber behavior becomes apparent at $U$ slightly less than that for the cloud with $N=10^{23} \mathrm{~cm}^{-2}$; however, the reduction in threshold $U$ is significantly less than the two orders of magnitude reduction in $N$. This disparity reflects the fact that the ionizing photon density that specifies $U$ is dominated by photons near the Lyman limit, while stripping of heavy elements is sensitive to the soft X-ray intensity. The apparent column density $N_{\text {eff }}$ will begin to deviate from $N$ when the soft X-ray flux is sufficient to produce highly ionized $O$ throughout the cloud. Clouds with $N \lesssim 10^{22} \mathrm{~cm}^{-2}$ will be optically thin to the continuum at $1 \mathrm{keV}$, regardless of ionization state (Morrison \& McCammon 1983); the X-ray continuum thus undergoes little attenuation, and substantial stripping of $\mathrm{O}$ will occur above a threshold $\mathrm{X}$-ray flux and hence $U$ that is independent of $N$. Increasing $N$ will result in a higher volume emission measure of line-emitting gas at a given $U$ but also will not produce a substantial relative increase in line emission in the warm absorber regime. For $N \gtrsim 10^{22}$ $\mathrm{cm}^{-2}$, the minimum $U$ for $N_{\text {eff }}$ variations begins to scale approximately in proportion to $N$, such that this threshold as well as the threshold for attaining a minimum $\operatorname{EW}(\operatorname{Ly} \alpha)$ will both increase as $N$ increases (cf. Figs. 3 and 2). In other words, the $U$ intervals corresponding to regimes of strong line emission and of warm absorber behavior will remain distinct.

A stronger association between line-emitting and warmabsorber material may result if the ionizing continuum is somewhat harder than the average spectral energy distribution employed heretofore. Increasing the ratio of soft X-ray to extreme ultraviolet photons will produce greater ionization of heavy elements at a given $U$ and hence will reduce the threshold $U$ for warm absorber behavior. This can be seen explicitly in Figure 6, which plots $\mathrm{EW}(\mathrm{Ly} \alpha)$ and $N_{\text {eff }}$ as a function of $U$ when the continuum is described by a power-law, $f_{v} \propto v^{\alpha}$ with $\alpha=-1.2$, changing to $\alpha=2.5$ longward of $1 \mu \mathrm{m}$ and $\alpha=-2$ at energies above $50 \mathrm{MeV}$. The cloud is again described by $n=10^{11} \mathrm{~cm}^{-3}$ and $N=10^{21} \mathrm{~cm}^{-2}$. The hotter nebula in this case produces slightly less Ly $\alpha$ emission (Fig. $6 a$ ), although the relative strength of collisionally excited lines increases. The behavior of $N_{\text {eff }}$ changes dramatically, entering the warm absorber regime at a lower threshold of $\log U \gtrsim-1$. If the covering factor of the warm-absorber medium is high, this material could thus contribute $\mathrm{EW}(\mathrm{Ly} \alpha)$ of up to several tens of angstroms. Emission in lines such as $\mathrm{C}$ IV and $\mathrm{N} v$ remains strong relative to $\mathrm{Ly} \alpha$ for $\log U \lesssim-0.5$, while $\mathrm{O}$ VI and Ne viII persist to $\log U \lesssim 0.5$.

Warm absorbers may thus produce significant line emission under some circumstances. Whether these conditions occur in many AGNs remains unclear, based on existing observational constraints. Additional information is needed on the typical covering factor, column density, and ionization state of this 


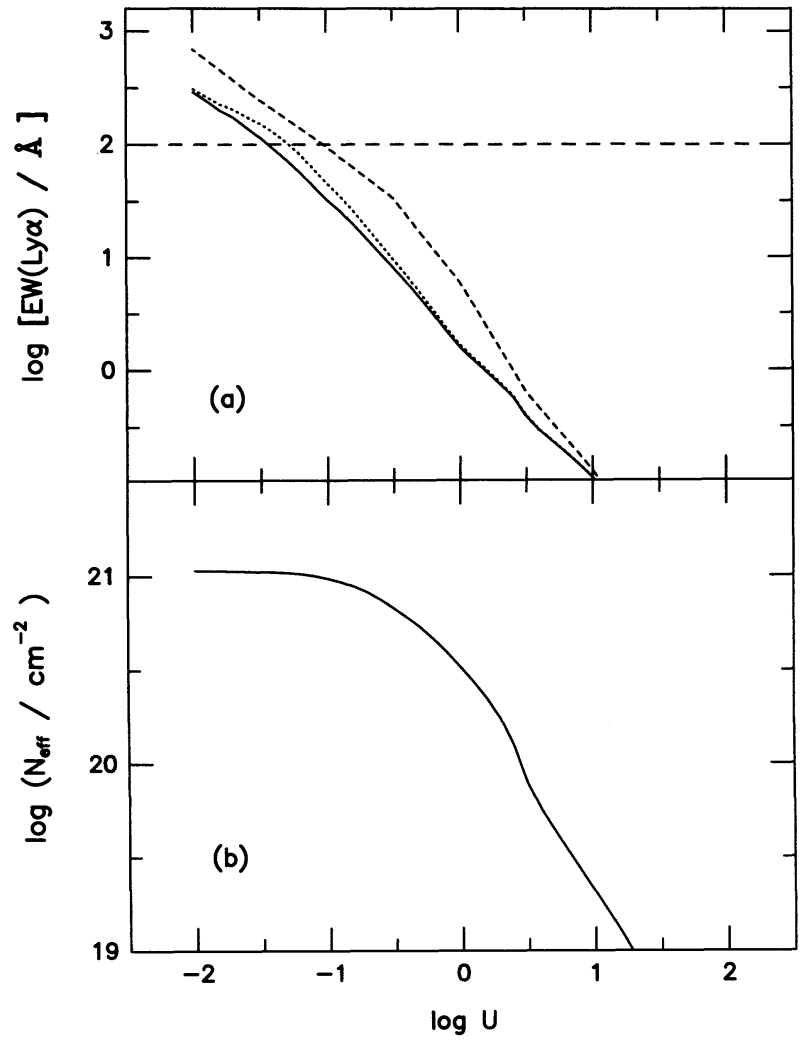

FIG. 6.-Same as Fig. 3, but for clouds irradiated by a power-law continuum with $\alpha=-1.2$.

medium. Recent studies of Fe K-edge absorption in AGNs imply that this feature is usually described by an energy of $\sim 8-9 \mathrm{keV}$ and depth corresponding to $N \sim 10^{23} \mathrm{~cm}^{-2}$ for solar abundances (Nandra \& Pounds 1994). While this column density may signal a large volume emission measure of gas, the energy of the absorption feature implies a typical ionization state of Fe XIX or higher (Makishima 1986); a medium this highly ionized is almost certainly an inefficient emitter of observable UV lines. Additional X-ray observations with good spectral resolution are desirable for confirmation of this result. More detailed information on the ionization state of the absorbing medium might be available in many cases from UV absorption studies. Mathur (1994) and Mathur et al. (1994) have recently identified two QSOs in which the absorption characteristics at UV and X-ray energies are consistent with a single medium along the line of sight. As described in $\S 2.2$, the ionization state of this material can be delimited from measurement of absorption lines spanning a range of ionization.

After this paper was accepted for publication, we became aware of recent $A S C A$ observations of three Seyfert nuclei reported by Fabian et al. (1994; MCG 6-30-15), Mihara et al. (1994; NGC 4051), and Ptak et al. (1994; NGC 3227). Their results show significant detections of absorption that can be attributed to $\mathrm{O}$ VII and $\mathrm{O}$ VIII, consistent with expectations for a warm absorber. MCG 6-30-15 and NGC 3227 exhibit temporal variation in the $\mathrm{X}$-ray continuum and absorption features that cannot be interpreted in terms of a simple absorber with ionization state closely tied to the ionizing radiation field. Similar behavior may also be present in BBXRT observations of NGC 4151 (Weaver et al. 1994). These findings may suggest that $n$ for the absorber is sufficiently low that its recombination timescale exceeds the timescale of variability in the X-ray continuum, i.e., the ionization of the absorbing plasma is not in equilibrium with the radiation field. If this is the case, the absorbers in these objects probably reside outside the BLR at a radial distance dictated by constraints on $U$ and $n$ (see $\S 2.1$ ). Alternatively, the absorbers might be located on smaller radial scales if they are actually multiphase systems (in terms of $n, N$, and $U$ ) that show a complex response in aggregate ionization to continuum variations. Recent observations of NGC 5548 with ROSAT demonstrate temporal variations in absorption indicating that an ionized absorber in this object has $n \gtrsim 5$ $\times 10^{4}$, implying that the absorber resides within $0.2 \mathrm{pc}$ of the central continuum source (Done et al. 1994).

\section{EVIDENCE FOR OPTICALLY THIN BLR CLOUDS: SPECIFIC CASES}

In the following sections we review observations of two wellstudied Seyfert galaxies in order to assess the possible significance of emission contributions from fully ionized clouds. In particular, we examine aspects of emission-line variability that may bear the imprint of optically thin nebular components, and the consistency of this interpretation with X-ray observations.

\subsection{Fairall 9}

Given the significance of Fairall 9 for motivating earlier suggestions of optically thin nebular behavior in BLRs, we have reexamined published line measurements for this source taken from Clavel et al. (1989). In order to examine possible saturation of emission-line luminosity we reviewed the line versus continuum behavior for $C_{\text {IV }}$ and $L y \alpha$ after removal of a time-delay appropriate for line response in this object. Based on the published data, we derived lags of 278 days and 238 days for $C$ IV and $L y \alpha$, respectively, relative to $1338 \AA$ continuum flux, using the cross-correlation technique described by White \& Peterson (1994). These numbers are larger than estimates of 200 and 115 days, respectively, obtained by Clavel et al. (1989). However, the line versus continuum crosscorrelation functions for these data are very broad, and the resulting lag estimates are consequently very uncertain; we derived formal error estimates of order 100 days.

Removal of a lag defined by the peak of the cross-correlation function corresponds to maximizing the correlation between line and continuum, and minimizing scatter when the two are plotted against each other. This can be seen in Figure 7, which shows the original data for Fairall 9 as observed and also after removal of a time delay $\tau_{\text {Iag }}$; i.e., each line measurement at time $t$ is referred to a continuum value at time $t-\tau_{\text {lag }}$. In this case we have adopted a single value of $\tau_{\text {lag }}=238$ days for both lines. Continuum flux levels appropriate to the line measurements at the time of observation were derived by interpolation from earlier data.

The apparent curvature or saturation in C IV noted by Wamsteker \& Colina (1986) is evident in Figure $7 a$ prior to removal of the lag. Figure $7 b$ shows the results for $C$ IV after removal of the lag between line and continuum measurements. An abrupt change of slope in emission-line response to the continuum is no longer evident, although significant curvature remains, leading to a flattening of line response at high continuum levels. Use of a lag of 278 days for $C$ IV results in the loss of the two points with the highest continuum flux, since the appropriate epoch for measurement of the continuum predates the earliest available data; this modification further reduces the 


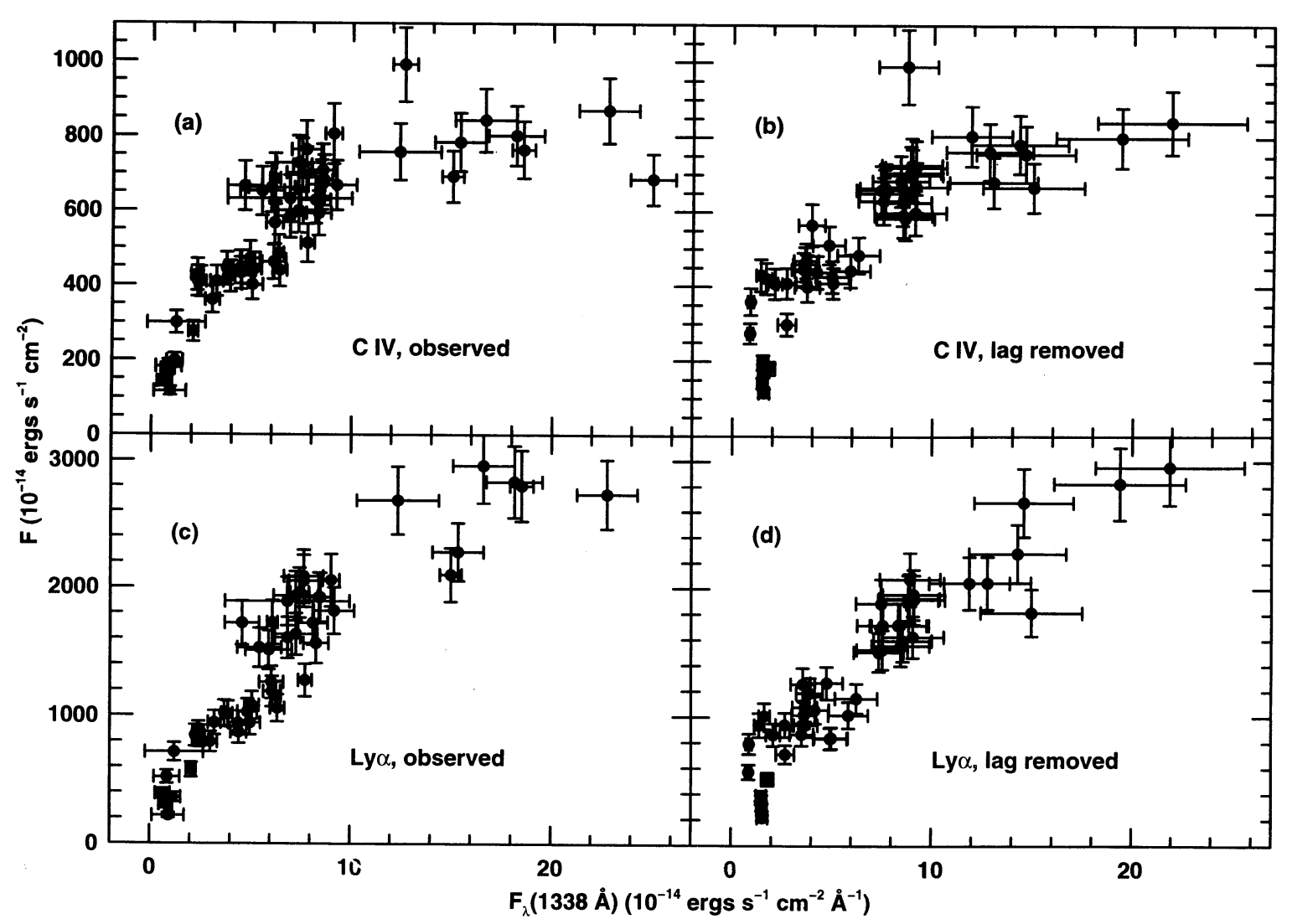

FIG. 7.-Line flux as a function of $1338 \AA \AA$ continuum flux measured for Fairall 9, before and after removal of a 238 day lag, for C IV $(a$ and $b)$ and Ly $\alpha(c$ and $d)$ The data and plotted observational uncertainties are taken from Clavel et al. (1989).

impression of saturation at high flux levels, although some curvature remains in the points at lower luminosity. The diagram for Ly $\alpha$ as observed (Fig. 7c) shows less evidence of saturation at high levels than does C IV, yet exhibits other, more complicated deviations from linear response. The response of $L y \alpha$ to the continuum after removal of the lag (Fig. 7d) shows evidence of curvature that is relatively weak except at the lowest flux levels.

In summary, an abrupt transition to a "saturated" state is not evident in either line after removal of the lag. However, it also appears difficult to describe the response of either line as proportional to the continuum by a constant factor, with any choice of $y$-intercept. Physically a positive $y$-intercept could represent a nonvariable (or slowly varying) component of line emission. Such a component would lead to an inverse relation between total line equivalent width and continuum luminosity, if the variable flux remains proportional to the continuum. If no constant term is present, the equivalent width of a line responding linearly to continuum changes should remain invariable.

The observational results presented here should be treated with caution. As can be seen in Figure 7, the measurement errors are often substantial. In addition, intervals between successive observations in this dataset vary widely, and the intrinsic variability is very likely undersampled in many instances. Interpolated estimates of the continuum referred to the measured line values may thus be in error by more than the bounds shown in Figure 7, which are derived from formal measurement uncertainties. Furthermore, removal of time-delay effects by a simple phase shift of one of the time series is an approx- imation that may or may not be valid, depending on the width and structure of the transfer function. As noted previously, estimates of $\tau_{\text {lag }}$ are also highly uncertain. Finally, it should be noted that the lag itself may vary between different episodes of variability, such that a single value for the time delay is not well defined (Netzer \& Maoz 1990).

We tentatively conclude that the behavior of C IV and Ly $\alpha$ emission in response to continuum changes in Fairall 9 can be interpreted in terms of optically thin nebular behavior, in agreement with conclusions by Wamsteker \& Colina. The observed pattern can be understood in terms of a population of clouds spanning a range of column density such that an increasing fraction of the BLR is optically thin at higher luminosity. The weaker sense of saturation seen in the Ly $\alpha$ versus continuum flux plot contrasted to the same figure for C IV might be due in part to increased $\mathrm{He} I \mathrm{II}$ and $\mathrm{O}$ v] contributions from optically thin plasma contributing to apparent $L y \alpha$ in high-luminosity states. Data streams with reduced uncertainties and improved sampling are desirable for confirmation of these conclusions.

If we dismiss the preceding evidence for optically thin BLR clouds in Fairall 9 as ambiguous or insubstantial, we are left with an unresolved puzzle of explaining the negative correlation between the $\mathrm{C}$ IV/Ly $\alpha$ ratio and continuum luminosity. The appearance of this ratio as a constant or decreasing function of continuum level is apparently a common occurrence in variable Seyferts (e.g., Kinney, Rivolo, \& Koratkar 1990; Pogge \& Peterson 1992; Gondhalekar 1992). Time delay analyses suggest further that to first order we can treat emission in these lines as originating in a common region (Clavel et 
al. 1989, 1991). Existing single-zone photoionization calculations predict, however, that $\mathrm{C}$ IV $/ \mathrm{Ly} \alpha$ should remain constant or increase as the ionizing continuum brightens (e.g., Ferland \& Persson 1989). The lack of agreement between theory and observation for these two energetically significant lines suggests that we may need to examine critically our assumptions concerning the nature of the BLR clouds and incident radiation field.

The C IV/Ly $\alpha$ ratio is sensitive to the ionizing spectral energy distribution (SED), and several authors have suggested that the observed response of the ratio to continuum fluctuations implies that the variable EUV continuum is dominated by a soft component (Binette et al. 1989; Clavel \& Santos-Lleó 1990; Gondhalekar 1992). In this scenario, continuum variations produce substantial changes in hydrogen ionization while introducing comparatively modest changes in carbon ionization and heating that control the $\mathrm{C}$ Iv luminosity. Since the EUV continuum eludes detection, direct constraints on this picture are lacking. However, the continuum SED can be constrained by methods analogous to Zanstra temperature determinations in $\mathrm{H}$ II regions, using the observed equivalent width of Ly $\alpha$ and possibly other lines. For at least some Seyfert nuclei exhibiting an inverse relation between $\mathrm{C}$ IV/Ly $\alpha$ and luminosity, including Fairall 9, observed $\operatorname{Ly} \alpha$ equivalent widths preclude a soft cutoff to the EUV continuum (Shields \& Ferland 1993). Extrapolation of the soft X-ray continuum observed in Fairall 9 is also inconsistent with a very soft EUV spectral distribution (Walter et al. 1994). A general understanding of the $\mathrm{C} I \mathrm{~V} / \mathrm{Ly} \alpha$ behavior in terms of soft EUV variation thus appears problematic.

Allowance for a mix of thin and thick clouds within the BLR could be the key to understanding the $C_{\text {IV }} /$ Ly $\alpha$ variation. As can be seen in Figure 1, the $\mathrm{C}$ IV/Ly $\alpha$ ratio is very high for a fully ionized cloud at intermediate $U$. For a composite BLR containing both thin and thick clouds, the thin clouds may strongly influence the composite spectrum while the continuum is in a low state. As the continuum brightens, the emission measure of the thick clouds will grow linearly while that of the thin clouds will remain constant. The composite spectrum will thus become increasingly weighted by emission from the thick cloud population, which is described by a lower C IV/Ly $\alpha$ emission ratio than that produced by the thin clouds. The $\mathrm{C}$ IV/Ly $\alpha$ ratio will then decrease as the continuum brightens, in qualitative agreement with the observational trend. We consider this possibility further in the following section, in a detailed comparison with observations of NGC 5548.

While this explanation resembles the scenario advanced by Wamsteker \& Colina (1986) by invoking fully ionized clouds, the two pictures differ in that Wamsteker \& Colina envisioned a global transition of the BLR to matter-bounded conditions, while we suggest that only a fraction of the BLR is matterbounded. The distinction is important, since in a global transition the emission becomes dominated by the thin clouds and the spectrum should be described by higher ionization and $\mathrm{C}$ IV/Ly $\alpha$ ratio in higher luminosity states, in contrast to the expectations for a mixed cloud population described above as well as the empirical spectral behavior.

X-ray emission from Fairall 9 studied by Morini et al. (1986) shows indications of a negative correlation between flux level and absorbing column density, which may provide direct evidence for thin clouds in the BLR acting as a warm absorber The typical inferred column density in this source is $N=10^{20}$ $10^{21} \mathrm{~cm}^{-2}$. The spectral index $\alpha_{o x}$ for Fairall 9 has varied between approximately -1.2 and -1.4 (Clavel et al. 1989), which is comparable to or somewhat harder than the average for AGNs. The variations in absorption are only apparent at very low energies $(\sim 250 \mathrm{eV})$ however, indicative of ionization changes in helium and possibly hydrogen rather than stripping of heavier elements. As a result, the absorption may result from material subject to considerably lower $U$ than for the case of variable $1 \mathrm{keV}$ absorption discussed in $\S 2.3$. A lack of $\mathrm{O}$ VIII $\mathrm{Ly} \alpha$ and $\operatorname{Ly} \beta$ emission in soft X-ray data reported by Lum et al. (1990) for this object is consistent with a characteristically low ionization state for this gas. Such a medium may generate a significant fraction of the observed BLR emission at UV wavelengths if the covering factor is high, and a direct identification of this medium with that responsible for the Wamsteker-Colina effect appears plausible (cf. Fig. $8 b$ for predicted behavior of $\mathrm{C}$ IV emission from a cloud with $N=10^{21}$ $\mathrm{cm}^{-2}$ ).

Since the X-ray-absorbing material is known to lie along the line of sight to the central engine, it is perhaps surprising that no absorption in ultraviolet resonance lines is evident in the IUE data for Fairall 9 (Ulrich 1988). The absence of obvious absorption may indicate that the $b$ values characteristic of the thin clouds in this source are small. However, an ultraviolet spectrum of Fairall 9 covering the C IV $\lambda 1549$ region with improved spectral resolution and signal-to-noise ratio, acquired with the Hubble Space Telescope in 1993, also does not show obvious C IV absorption (M. Crenshaw, private

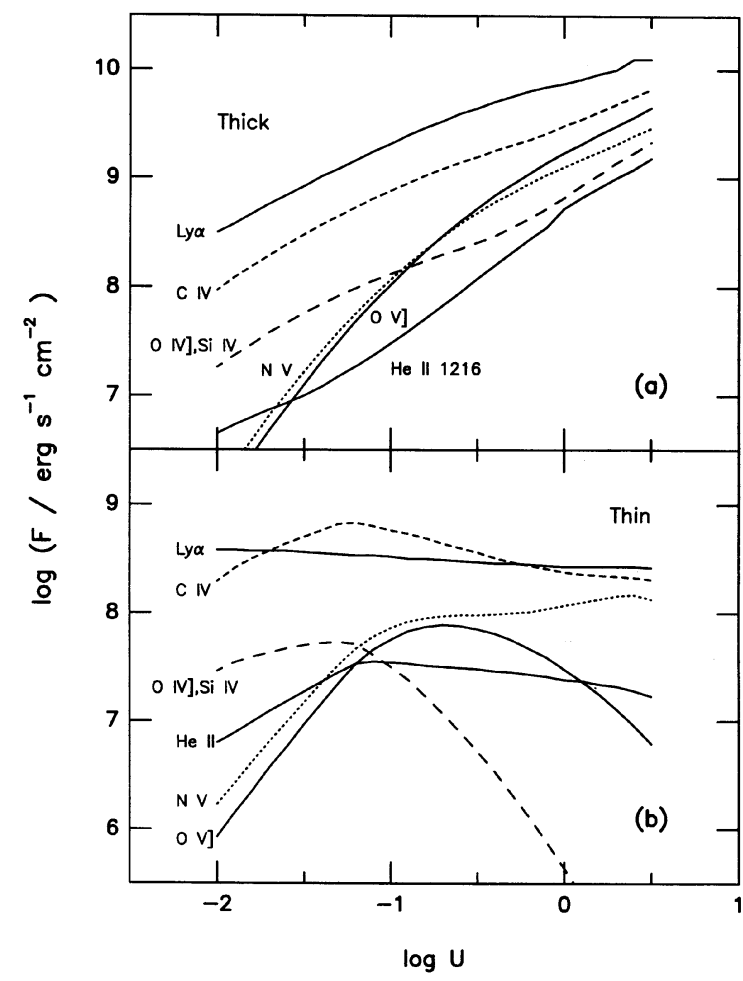

FIG. 8.-Predicted emission-line fluxes as a function of $U$, for clouds with $n=10^{11} \mathrm{~cm}^{-3}$, irradiated by the average AGN continuum. The top panel assumes ionization-bounded clouds, while the bottom panel assumes $N=10^{21}$ $\mathrm{cm}^{-2}$. Line profiles for the optically thin medium are broadened by $b_{\text {turb }}=100$ $\mathrm{km} \mathrm{s}^{-1}$. Variations in He II $\lambda 1640$ parallel those shown for $\lambda 1216$ (cf. Fig. 1a). 
communication), although some absorption could be present in the rather complex $\mathrm{C}$ IV emission peak. A lack of absorption could result if the ionization state of the gas has evolved so that little $\mathrm{C}$ IV is present in the thin cloud, if the line-of-sight coverage has changed since the X-ray data were acquired in 1984 (Morini et al. 1986), or if the spatial distribution of the ultraviolet and $\mathrm{X}$-ray continuum sources is sufficiently different that line-of-sight coverage need not correspond for the two bandpasses.

\subsection{NGC 5548}

Intensive monitoring of the Seyfert 1 galaxy NGC 5548 has led to a large database of ultraviolet and optical emission-line measurements for a range of luminosity states. One result of this campaign is a cross-correlation analysis of line response to the continuum that provides direct evidence for optically thin nebular components in the inner BLR, as noted in $\S 1$ (Sparke 1993). The observational data have also permitted quantification of emission-line response to continuum variations in terms of slope (Krolik et al. 1991; Pogge \& Peterson 1992) and characteristic time delays (see Peterson 1993 for a summary). NGC 5548 shows significant deviations from linearity in the response of emission-line flux to changes in the ultraviolet continuum. One manifestation of the observed behavior is an inverse correlation between $\mathrm{C}$ IV $\lambda 1549 / \mathrm{Ly} \alpha$ intensity ratio and continuum luminosity, as in Fairall 9. Krolik et al. (1991) have published a model for NGC 5548 employing two populations of photoionized clouds, which reproduces many of the gross characteristics of this source but predicts differential responses in Ly $\alpha$ and C IV that are inconsistent with the observations by more than $6 \sigma$ and $11 \sigma$, respectively. The predicted luminosity dependence of the $\mathrm{C}$ IV/Ly $\alpha$ ratio once again disagrees with the observations, not simply in terms of amplitude, but in sign (i.e., a positive correlation is predicted).

NGC 5548 presents another case in which a mix of thick and thin clouds in the BLR might provide a means of understanding the $\mathrm{C}$ IV/Ly $\alpha$ behavior. The extensive base of quantitative data for this object allows us to go beyond qualitative explanations, however, and enables a detailed test of the current scenario. A quantitative assessment of the thick + thin cloud picture is highly desirable in light of several basic questions concerning its viability. The equivalent width of thin cloud emission declines rapidly with increasing luminosity (Figs. 2 and 3); can sufficient emission be generated to influence the total spectrum? If so, are the required covering factors for thin and thick components reasonable? Is it possible to reproduce the behavior of $\mathrm{C}$ IV and Ly $\alpha$ in terms of logarithmic slopes as well as equivalent widths? If the $\mathrm{C}$ IV/Ly $\alpha$ behavior is matched to the observations, does this introduce problems in explaining the ratios and differential behavior for other high ionization lines?

In an effort to address these issues, we constructed a twocomponent scenario for the inner BLR of NGC 5548 consisting of optically thick and thin clouds with uniform $n=10^{11}$ $\mathrm{cm}^{-3}$. The continuum was represented by the standard AGN SED, as described in $\S 2$. Calculations for the thick component were stopped at the depth resulting in the hydrogen ionization fraction falling below 0.6 ; at $\log U=0$, this corresponds to $N=5 \times 10^{23} \mathrm{~cm}^{-2}$. The thin component was assumed to have a uniform column density of $10^{21} \mathrm{~cm}^{-2}$, which leads to full ionization at $\log U \gtrsim-2.5$. We additionally assumed that the line profiles in the thin medium were modified by a velocity field represented by $b_{\text {turb }}=100 \mathrm{~km} \mathrm{~s}^{-1}$, which is considerably less than the observed width of the broad lines in this object $\left(\sigma \approx 2000-4000 \mathrm{~km} \mathrm{~s}^{-1}\right.$; Krolik et al. 1991).

Line fluxes emitted by the thick and thin components and relevant for comparison with the observations are plotted as functions of $U$ in Figure 8. The thick component exhibits an initially steep rise in the strength of $\mathrm{N} \mathrm{v}$ and $\mathrm{O} v$ ], but an approximately linear response in the other plotted lines, with nearly constant relative intensities at large $U$. In contrast, the fully ionized thin component produces a nearly constant Ly $\alpha$ flux, He II emission that initially grows and then saturates as helium becomes fully ionized, and emission in other lines that peaks at intermediate $U$, reflecting the changing ionization state of the heavy elements. Agreement with many aspects of emission-line behavior in NGC 5548 results for a composite spherical system consisting of thin clouds with covering factor 0.45 , and thick clouds with $f_{c}=0.15$ subject to the same $U$. The results, in terms of relative line strengths and slopes with respect to $U$, are listed in Table 1 for $-1.5 \leq U \leq-0.5$. Line fluxes are normalized to the Ly $\alpha$ blend, consisting of contributions from $\mathrm{Ly} \alpha \lambda 1216, \mathrm{He}$ II $\lambda 1216$, and $\mathrm{O} v \mathrm{v}] \lambda 1218$. The differential behavior of the blended feature and its equivalent width are also listed. Corresponding parameters for broad lines measured in NGC 5548 and tabulated by Krolik et al. (1991) are listed for comparison. The empirical values were derived over an interval in which the $1360 \AA$ continuum flux from the nucleus of NGC 5548 varied by a factor of $\sim 3.5$.

Predictions for the composite system are successful in reproducing the observed equivalent widths and relative fluxes of

TABLE 1

Composite Emission-LiNe FluXes

\begin{tabular}{|c|c|c|c|c|c|c|}
\hline $\begin{array}{l}\text { Predicted Relative Fluxes: } \\
-1.5 \ldots \ldots \ldots \ldots \ldots \ldots \ldots \ldots \ldots \\
-1.0 \ldots \ldots \ldots \ldots \ldots \ldots \ldots \ldots \ldots \\
-0.5 \quad \ldots \ldots \ldots \ldots \ldots \ldots \ldots \ldots\end{array}$ & $\begin{array}{r}214 \\
115 \\
66\end{array}$ & $\begin{array}{l}1 \\
1 \\
1\end{array}$ & $\begin{array}{l}0.030 \\
0.098 \\
0.123\end{array}$ & $\begin{array}{l}0.90 \\
0.73 \\
0.42\end{array}$ & $\begin{array}{l}0.10 \\
0.13 \\
0.13\end{array}$ & $\begin{array}{l}0.032 \\
0.066 \\
0.043\end{array}$ \\
\hline NGC $5548:^{c} \ldots \ldots \ldots \ldots \ldots$ & 160 & 1 & 0.12 & 0.85 & 0.12 & 0.10 \\
\hline NGC $5548:^{c} \ldots \ldots \ldots \ldots \ldots$ & $\cdots$ & $0.51 \pm 0.06$ & $1.29 \pm 0.24$ & $0.35 \pm 0.06$ & $0.91 \pm 0.21$ & $0.48 \pm 0.11$ \\
\hline
\end{tabular}

a Including $\mathrm{Ly} \alpha \lambda 1216, \mathrm{He}$ II $\lambda 1216$, and $\mathrm{O}$ v] $\lambda 1218$.

b He II $\lambda 1640$.

c Mean values taken from Krolik et al. 1991. 
the high ionization lines, for $\log U$ varying from approximately -1.5 to -1.0 . The corresponding logarithmic slope of line versus continuum flux is in reasonable agreement with observed values for the Ly $\alpha$ blend, N v, C IV, and He II. The $\mathrm{C}$ IV/Ly $\alpha$ intensity ratio decreases with increasing luminosity in a manner consistent with observation. The ratio for the thick cloud is consistently less than for the thin cloud over the $U$ interval of interest, resulting in a decrease in the composite ratio with increasing $U$ as the fractional luminosity from the thick component grows.

The calculations presented here are intended to be illustrative of the effects of a heterogeneous BLR cloud population, and provide direction for future comprehensive models of the BLR that are beyond the scope of the current study. The quantitative comparison with NGC 5548 demonstrates the viability of employing a composite cloud population to represent the BLR, but should not be taken as a definitive description of this object. Examination of Table 1 shows, for example, that the predicted differential response of $\mathrm{Si}$ IV $+\mathrm{O}$ IV] $\lambda 1400$ is considerably weaker than observed. We emphasize that predictions of the relative response of different lines in this two-component picture are highly sensitive to choices of $U, n$, and $f_{c}$ for each of the two components and $N$ and $b_{\text {turb }}$ for the thin clouds, as well as SED of the incident continuum (which may be radially dependent due to foreground shadowing).

The present combination of calculation parameters was selected in order to optimize agreement with the observations while minimizing fine-tuning. We thus consider it highly probable that agreement between the predicted and observed response for $\mathrm{Si}$ IV $+\mathrm{O}$ IV] can be improved with suitable adjustment of these parameters. Attaining such agreement is not necessarily physically meaningful, however, since representation of the emitting plasma by two uniform cloud populations is undoubtedly a gross simplification. In reality this nebulosity should probably be described by a distribution function of clouds as a function of $n, N$, and radial location. Moreover, the cloud components considered here are only relevant for describing the inner BLR. Emission in C III] $\lambda 1909$ and lines from other low-ionization species is substantially underpredicted for these clouds, consistent with time-delay measurements implying that these features originate at characteristically larger radii. A comprehensive description of the BLR in NGC 5548 addressing all of these considerations is the subject of work in progress that builds on recent spectroscopic monitoring of this source by the Hubble Space Telescope (Korista et al. 1995).

The ultraviolet spectrum of NGC 5548 exhibits resonance absorption features that have been studied in detail by Shull \& Sachs (1993). They reported an inverse correlation between the equivalent width of $\mathrm{C}$ IV absorption and continuum flux most readily interpreted as a decrease in $C$ IV column density with increasing $U$. This behavior would be consistent with predictions for our thin cloud component for $\log U \geq-1.5$ (Fig. 5); $\mathrm{Mg}$ II absorption is likely to be negligible in this regime, consistent with its absence in the observations. The observed velocity width of C IV absorption is of order $\sim 3000 \mathrm{~km} \mathrm{~s}^{-1}$. Shull \& Sachs derived a lower limit to the $C$ IV column density in the absorbing medium of $1.3 \times 10^{15} \mathrm{~cm}^{-2}$; this bound is consistent with the thin medium considered here for $\log U \lesssim 0.5$. An identification of the absorbing material with the thin BLR component contributing line emission as discussed above thus appears reasonable.

NGC 5548 exhibits evidence for an X-ray-absorbing column with $N \approx 10^{21}-10^{22} \mathrm{~cm}^{-2}$, and a weak tendency for absorbing columns to be larger at lower $2-10 \mathrm{keV}$ flux levels, qualitatively consistent with the warm absorber model (Nandra et al. 1991). Evidence for an absorption edge resulting from highly ionized oxygen has also been reported (Nandra et al. 1993). In the emission-line analysis discussed above for this source, the average AGN SED was employed for reasons of simplicity. For photoionization by such a continuum, significant line emission from the warm absorber is unlikely. Direct observations suggest that the continuum in NGC 5548 is probably somewhat harder than the generic continuum, however, with $\alpha_{o x} \approx-1.2$ (Clavel et al. 1992). (The X-ray continuum itself features $\alpha \approx-0.8$; Nandra et al. 1991.) As discussed in $\S 2.3$, irradiation by a harder continuum of this type increases the likelihood that the warm absorber could generate observationally significant line emission.

The photoionization calculations employing $\alpha=-1.2$ and $N=10^{21} \mathrm{~cm}^{-2}(\S 2.3)$ also predict predominant states of iron within the absorber to be Fe IX-Fe XV for $\log U=-1.0-0.0$, which would produce K-edge absorption at 7.3-7.6 keV and $\mathrm{K} \alpha$ emission at $6.4 \mathrm{keV}$ (Makishima 1986), consistent with a probable absorption edge at 7-8 keV and emission at $6.3 \pm 0.1$ $\mathrm{keV}$ reported by Nandra et al. (1991) for NGC 5548. In the limit of $f_{c}=1$, the predicted equivalent width of $\mathrm{Fe} \mathrm{K} \alpha$ emission at $\log U=0$ is only $\sim 3 \mathrm{eV}$, however, much less than the observed value of $130 \pm 40 \mathrm{eV}$ (Nandra et al. 1991). Increasing $N$ to $10^{22} \mathrm{~cm}^{-2}$ would provide somewhat better agreement with the strength of the observed absorption edge and would increase the equivalent width of $\mathrm{Fe} \mathrm{K} \alpha$ to $\sim 30 \mathrm{eV}$, but this value is still well below the measured equivalent width. Additional emission from other components is probably necessary to produce the total observed $\mathrm{Fe} \mathrm{K} \alpha$. Production of $\mathrm{Fe} \mathrm{K} \alpha$ in high- $N$ clouds with substantial covering factor appears to be a general characteristic of Seyfert 1 nuclei, based on observed equivalent widths for this feature (e.g., Nandra et al. 1989; Matsuoka et al. 1990).

We note that use of a harder continuum leads to stronger emission in cooling ultraviolet transitions such as the $\mathrm{C}$ IV and $\mathrm{N} v$ features, due to the increased heating per ionization. Representing the continuum in NGC 5548 by a power-law continuum as in the present discussion, rather than the generic AGN continuum, will thus introduce changes in predicted line fluxes and require modification of the formulation employed in Table 1 to match emission line behavior. Attempts to produce a simple two-component representation as before, but using the power-law continuum, were unsuccessful due to excessive strength of the collisionally excited lines. We suspect that filtering of the continuum by an inner thin cloud layer may be important for modifying the radiation field seen by clouds at larger radii, such that the aggregate emitted spectrum displays weaker heavy-element line emission than our calculations would suggest.

\section{THE BALDWIN EFFECT}

Ensembles of AGNs show a nonlinear relation between emission-line and continuum luminosity that is qualitatively similar to the behavior seen in individual, variable sources (Wamsteker \& Colina 1986; Kinney et al. 1990; Pogge \& Peterson 1992), although the deviation from linearity is less for the ensemble case. This behavior is usually expressed as a trend of decreasing emission-line equivalent width with increasing luminosity (i.e., the "Baldwin effect;" Baldwin 1977). Decreasing equivalent widths could be explained in terms of a system- 
atic decrease in $f_{c}$ with increasing source luminosity (e.g., Wu, Boggess, \& Gull 1983). However, the strength of the decrease in equivalent width differs for different lines, such that line ratios show systematic trends with luminosity. In particular, increasing luminosity is accompanied by decreasing $\mathrm{C}$ IV/Ly $\alpha$ and increasing C III]/C IV (e.g., Baldwin, Wampler, \& Gaskell 1989; Kinney et al. 1990); there also appears to be some tendency for $\mathrm{O} \mathrm{vI} / \mathrm{Ly} \alpha$ to be smaller in more luminous sources (cf. Wilkes 1984; Kinney et al. 1991; Kriss et al. 1992; Zheng et al. 1992).

The systematic behavior of line ratios has motivated efforts to explain the Baldwin effect in terms of luminosity-dependent parameters other than simply $f_{c}$. Possibilities include scenarios in which increasing luminosity is accompanied by decreasing $U$ (Mushotzky \& Ferland 1984) or softening ionizing continuum shape (Schultz 1992); decreasing characteristic cloud density might also be consistent (Rees, Netzer, \& Ferland 1989). The general trend appears as a decrease in nebular ionization or excitation with increasing luminosity. An exception to this statement is seen in $\mathrm{N} \mathrm{v}$, which tends to increase significantly in strength with increasing continuum luminosity (e.g., Osmer, Porter, \& Green 1994); this behavior is likely to be an abundance effect, however (Hamann \& Ferland 1992, 1993). While changes in $U$, continuum shape, and/or cloud density with source luminosity may help explain the Baldwin effect and associated trends in line ratios, it is important to note that a luminosity-dependent $f_{c}$ may be indicated independently by a negative correlation between soft-X-ray-absorbing column density and luminosity for AGNs (Lawrence \& Elvis 1982).

The present work on emission-line variability in Seyfert nuclei suggests another variant of explanations for the Baldwin effect that provides a physical link between nonlinearity of line response in ensembles (the "global" Baldwin effect) and in individual sources (the "intrinsic" Baldwin effect; Pogge \& Peterson 1992). As demonstrated in $\S 3$, the nonlinearity of line response to continuum luminosity, and the $\mathrm{C}$ IV/Ly $\alpha$ behavior in particular, can be understood in individual variable objects as a consequence of thin, matter-bounded clouds within the BLR. At a given $U$, the thin clouds produce stronger emission in the high ionization lines than the thick BLR clouds, but as $U$ increases, the relative importance of the thin component for producing line emission diminishes. If the covering factor of thin clouds systematically decreases with luminosity in the AGN ensemble, their importance for generating line emission will similarly decrease and produce changes in equivalent width and line ratios that may be consistent with the global effect.

For a spherically symmetric distribution of clouds, the detailed dependence of line emission on central engine luminosity $L$ will depend on the radial distribution of clouds and their column densities, since the radius of constant $U$ for fixed $n$ will grow as $L^{1 / 2}$. If the covering factor and proportion of thick and thin clouds are constant as a function of radius, emission from clouds at small radii decreases in importance as $L$ increases and $\log U$ exceeds $\sim 0$ : emission from thin clouds saturates due to their fixed emission measure, and emission from thick clouds saturates due to line destruction and electron scattering (Shields \& Ferland 1993). Emission in the highionization lines will thus result primarily from a shell that grows in radius as $L$ increases, and a homologous cloud distribution will lead to homologous spectra in terms of line ratios and equivalent widths. For a variable Seyfert nucleus, we can assume that the cloud distribution is fixed on short timescales so that the observed line ratio behavior suggests a decrease in $f_{c}$ of thin clouds with increasing radius. The global Baldwin relations could be accounted for by a similar radial distribution that does not scale in metric size with luminosity, or alternatively another mechanism leading to decreased $f_{c}$ with increasing $L$.

The explanation for the ensemble Baldwin effect described here is in some sense a synthesis of earlier interpretations involving luminosity-dependent $f_{c}, U$, and thin clouds. Explaining the global Baldwin effect in terms of a lower $f_{c}$ of thin clouds in more luminous sources is consistent in general terms with trends of decreasing X-ray absorption with increasing source luminosity. While invoking luminosity-dependent coverage, this scenario can also be viewed in some sense as a pattern of decreasing $U$ with increasing luminosity, since the thin clouds lack deep regions described locally by a low $U$, in contrast to the thick clouds. Finally, while fully ionized clouds play an important role in this picture, their importance for generating the total line emission of an AGN diminishes with increasing source luminosity, which is opposite the behavior envisioned by Wamsteker \& Colina (1986) in explaining the Baldwin effect by a global transition of the BLR to matterbounded conditions.

If this scenario is correct, what is the physical mechanism that leads to a reduction in coverage by thin clouds at high luminosity and/or large radius? Thin clouds observed in absorption in Seyfert nuclei tend to be blueshifted relative to the emission lines by up to $\sim 1000 \mathrm{~km} \mathrm{~s}^{-1}$, indicative of outflow (Ulrich 1988). This speed is considerably greater than the sound speed within the absorbing gas; if the clouds thus remain roughly constant in size as they move out in radius, a radial decrease in $f_{c}$ would be a natural consequence. In addition, if such clouds are more efficiently accelerated and ejected in more luminous nuclei, a reduction of $f_{c}$ with increasing luminosity would result. The presence of broad absorption line (BAL) features blueshifted by up to $\sim 30,000 \mathrm{~km} \mathrm{~s}^{-1}$ in luminous QSOs might provide direct evidence for this scenario; the coverage of the BAL clouds is also thought to be low on average, with $f_{c} \approx 0.1$ (Hamann, Korista, \& Morris 1993).

An interesting consistency with this picture appears in differences in behavior for radio-loud and radio-quiet QSOs. BAL features are found only in radio-quiet objects (Stocke et al. 1992). If the occurrence of BAL absorption is an indication of efficient removal of thin clouds, then we might expect radioquiet QSOs to be largely stripped of such material. If the removal of thin clouds in the radio-loud AGNs proceeds less efficiently, we might expect the radio-loud sources to exhibit stronger signatures of a thin-cloud BLR component when compared with radio-quiet objects. This scenario is consistent with observational evidence that the global Baldwin effect is stronger in radio-loud quasars than in radio-quiet QSOs (Steidel \& Sargent 1991), and that luminous radio-loud sources show greater soft-X-ray absorption than radio-quiet objects (Bechtold et al. 1994 and references therein). In terms of overall emission-line characteristics, Tytler \& Fan (1992) reported some tendency for radio-loud QSOs to exhibit larger equivalent widths of $\mathrm{C}$ IV, Si IV + O IV], and possibly Ly $\alpha$ that might be consistent with this expectation, although they noted that this trend could arise from a selection effect in their sample. Steidel \& Sargent (1991), however, found no statistically significant differences between the high ionization lines of radioloud and radio-quiet sources. If an extra component of high-ionization material is often present in radio-loud AGNs, 
the emission-line signatures of this plasma are evidently lost in many cases in the (large) spread of emission-line characteristics stemming from intrinsic variations in other AGN parameters.

Radio-loud QSOs preferentially show narrow absorption features from clouds at or near the redshift of the AGN (Foltz et al. 1988), which conceivably could be identified with an extra BLR component in radio-loud sources compared with radioquiet objects. Density estimates are required in order to constrain the radial location of the absorber ( $\$ 2.2)$. In the few cases of these "associated absorbers" studied in detail to date, the resulting estimates of distance from the central engine range from many kiloparsecs (Williams et al. 1975; Morris et al. 1986), to $\lesssim 200$ pc (Hamann et al. 1995). The absorbing entities are thus likely to be a heterogeneous class of object, and additional measurements are necessary to determine whether a significant fraction of these systems are related in some way to the BLR.

\section{CONCLUSIONS}

This paper presents the results of photoionization calculations that explore the behavior of clouds within the broadline region that become fully ionized in hydrogen. The presence of such clouds is suggested by several pieces of existing evidence. We find that a mix of thin (fully ionized) and thick (ionization-bounded) clouds may have a significant role in producing the observed nonlinear response of emission lines to the continuum in variable Seyfert galaxies, including the associated pattern of negative correlation between the $\mathrm{C}$ IV $/$ Ly $\alpha$ intensity ratio and luminosity. Thin, fully ionized clouds provide a means of understanding the high degree of symmetry observed in Ly $\alpha$ emission profiles, since such nebulae are isotropic line emitters and also may produce symmetrical broadening of profiles via electron scattering if column densities are sufficiently high.

Thin clouds lying along our line of sight to the central engine are expected to provide absorption signatures that are consistent with observed UV resonance-line absorption and soft X-ray attenuation by cold or partially ionized matter ("warm absorbers"). Warm absorber media are likely to be described by a high $U$ but may nonetheless contribute significant UV line emission, notably of $\mathrm{Ne}$ VIII $\lambda 774, \mathrm{O}$ VI $\lambda 1035$, and Ly $\alpha$; emission in other lines may also be nonnegligible if the ionizing continuum is relatively hard, as is often the case for Seyfert nuclei, and $U$ is not too high. Specific comparisons with observations of Fairall 9 and NGC 5548 suggest that thin clouds are likely to be important in determining the UV emission-line properties in both objects. For Fairall 9, an apparent saturation in $\mathrm{C}$ IV emission strength at high luminosity can be plausibly attributed to a fully-ionized BLR component, as originally suggested by Wamsteker \& Colina (1986), and the same medium may be responsible for X-ray absorption with variable soft-energy opacity seen in this source. For NGC 5548, a thin component responsible for significant line emission would also be capable of producing the observed UV absorption spectrum of this object.

The influence of thin clouds provides a common framework for understanding the nonlinear correlation of line and continuum emission in both variable Seyfert nuclei and AGN ensembles-i.e., the "intrinsic" and "global" Baldwin effects. In both cases, the behavior of line ratios and equivalent widths as a function of luminosity can be understood in terms of decreasing coverage of thin clouds at larger radii. The radial distribution of thin clouds and lack of such media in luminous AGNs may result if these tenuous components are blown out of the BLR with an efficiency that increases in more luminous sources. Such a mechanism is suggested by blueshifted absorption that is commonly seen in AGNs and which achieves its most extreme manifestation in BAL features in QSOs.

Improved understanding of the BLR and its larger relationship to the AGN phenomenon will result from observational probes of the thin cloud component and its inclusion in theoretical analyses. Thin clouds in the inner BLR will emit strong, high-ionization lines, and can be traced by emission in $\mathrm{O}$ VI and $\mathrm{Ne}$ VIII in particular. Ultraviolet and X-ray variability measurements will continue to have a major role in defining the characteristics of this material. Observations with high spectral resolution, e.g., with $H S T$ and $A S C A$, will be especially useful for studies of thin clouds seen in absorption, in order to determine the velocity distribution of absorbing clouds from UV resonance lines, and ionization state as revealed by soft X-ray absorption structure. Variability studies of Seyfert nuclei have clearly demonstrated that a single population of emitting clouds is not a good description of the BLR. The present work suggests that future BLR models would benefit from inclusion of clouds with a range of column density, leading to a mix of ionization-bounded and matterbounded structures.

This work was supported by NSF grant AST 90-19692 and NASA Long-Term Space Astrophysics grant NAGW-3315. J. C. S. also received support from NASA through grant number HF-1052.01-93A from the Space Telescope Science Institute, which is operated by the Association of Universities for Research in Astronomy, Inc., under NASA contract NAS526555. We thank M. Elvis, F. Hamann, K. Korista, S. Mathur, P. Osmer, B. Wilkes, and the referee for informative comments and discussions, and J. Krolik and R. Mushotzky for drawing our attention to recent $A S C A$ results.
Anderson, K. S. 1974, ApJ, 189, 195

Avrett, E. H., \& Loeser, R. 1988, ApJ, 331, 21

Baldwin, J. A. 1977, ApJ, 214, 679

Baldwin, J. A., Wampler, E. J., \& Gaskell, C. M. 1989, ApJ, 338, 630

Bechtold, J, et al 1994, AJ, 108, 759

Binette, L., Prieto, A., Szuszkiewicz, E., \& Zheng, W. 1989, ApJ, 343, 135

Bromage, G. E., et al. 1985, MNRAS, 215,

Bromage, G. E., et al. 1985, MNRAS,
Clavel, J., et al. 1991, ApJ, 366, 64

J., et al. 1991, ApJ, 366, ApJ, 393, 113

Clavel, J., \& Santos-Lleó, M. 1990, A\&A, 230, 3

Clavel, J., Wamsteker, W., \& Glass, I. S. 1989, ApJ, 337, 236

Collin-Souffrin, S., Dumont, S., Joly, M., \& Péquignot, D. 1986, A\&A, 166, 27

Davidson, K. 1977, ApJ, 218, 20

Davidson, K., \& Netzer, H. 1979, Rev. Mod. Phys., 51, 715

Done, C., Pounds, K. A., Nandra, K., \& Fabian, A. C. 1994, in Reverberation

Mapping of the Broad-Line Region in Active Galactic Nuclei, ed. P. M.

Gondhalekar, K. Horne, \& B. M. Peterson (ASP Conf. Ser., 69), 211

Emmering, R. T., Blandford, R. D., \& Shlosman, I. 1992, ApJ, 385, 460

\section{REFERENCES}

Fabian, A. C., et al. 1994, PASJ, 46, L59

Ferland, G. J. 1993, University of Kentucky Department of Physics \& Astronomy Internal Report

Ferland, G. J., Korista, K. T., \& Peterson, B. M. 1990, ApJ, 363, L21

Ferland, G. J., \& Persson, S. E. 1989, ApJ, 347, 656

Ferland, G. J., Peterson, B. M. Horne, K., Welsh, W. F. \& Nahar, S. N. 1992 ApJ, 387, 95

Fiore, F., Elvis, M., Mathur, S., Wilkes, B. J., \& McDowell, J. C. 1993, ApJ, 415,129

Foltz, C. B., Chaffee, F. H., Hewett, P. C., Weymann, R. J., \& Morris, S. L. 1990, BAAS, 22, 806

Foltz, C. B., Chaffee, F. H., Weymann, R. J., \& Anderson, S. F. 1988, in QSO Absorption Lines, ed. J. C. Blades, D. Turnshek, \& C. A. Norman Cambridge: Cambridge Univ. Press) 53

Gondhalekar, P. M. 1992, MNRAS, 255, 663

Grevesse, N., \& Anders, E. 1989, in Cosmic Abundances of Matter (AIP Conf. Proc. 183), ed. C. J. Waddington (New York: AIP), 1

Halpern, J. P. 1984, ApJ, 281, 90 
Hamann, F., Shields, J. C., Diplas, A., \& Ferland, G. J. 1994, ApJ, submitted Hamann, F., et al. 1995, ApJ, in press

Hamann, F., \& Ferland, G. J. 1992, ApJ, 391, L53

.1993, ApJ, 418,11

Hamann, F., Korista, K. T., \& Morris, S. L. 1993, ApJ, 415, 541

Kinney, A. L., Bohlin, R. C., Blades, J. C., \& York, D. G. 1991, ApJS, 75, 645

Kinney, A. L., Rivolo, A. R., \& Koratkar, A. P. 1990, ApJ, 357, 338

Kolman, M., Halpern, J. P., Martin, C., Awaki, H., \& Koyama, K. 1993, ApJ,

4.I 403, 592

Korista, K. T., et al. 1995, ApJS, in press

Korista, K. T., Voit, G. M., Morris, S. L., \& Weymann, R. J. 1993, ApJS, 88, 357

Kriss, G. A., et al. 1992, ApJ, 392, 485

Kriss, G. A., \& Canizares, C. R. 1985, ApJ, 297, 177

Krolik, J. H., Horne, K., Kallman, T. R., Malkan, M. A., Edelson, R. A., \& Kriss, G. A. 1991, ApJ, 371, 541

Krolik, J. H., \& Kallman, T. R. 1984, ApJ, 286, 366

Kwan, J., \& Krolik, J. H. 1981, ApJ, 250, 478

Laor, A., Bahcall, J. N., Jannuzi, B. T., Schneider, D. P., Green, R. F., \& Hartig, G. F. 1994, ApJ, 420, 110

Lawrence, A., \& Elvis, M. 1982, ApJ, 256, 410

Lum, S. K., Canizares, C. R., Markert, T. H., \& Arnaud, K. A. 1990, ApJ, 358, 139

Makishima, K. 1986, in The Physics of Accretion onto Compact Objects, ed. K. O. Mason, M. G. Watson, \& N. E. White (Berlin: Springer), 249

Mathews, W. G., \& Ferland, G. J. 1987, ApJ, 323, 456

Mathur, S. 1994, ApJ, 431, L75

Mathur, S., Wilkes, B., Elvis, M., \& Fiore, F. 1994, ApJ, 434, 493

Matsuoka, M., Piro, L., Yamauchi, M., \& Murakami, T. 1990, ApJ, 361, 440

Mihara, T., Matsuoka, M., Mushotzky, R. F., Kunieda, H., Otani, C., Miyamoto, S., \& Yamauchi, M. 1994, PASJ, 46, L137

Morini, M., et al. 1986, ApJ, 307, 486

Morris, S. L., \& Ward, M. J. 1989, ApJ, 340, 713

Morris, S. L., Weymann, R. J., Foltz, C. B., Turnshek, D. A., Shectman, S., Price, C., \& Boroson, T. A. 1986, ApJ, 310, 40

Morrison, R., \& McCammon, D. 1983, ApJ, 270, 119

Mushotzky, R., \& Ferland, G. J. 1984, ApJ, 278, 558

Nandra, K., et al. 1993, MNRAS, 260, 504

Nandra, K., \& Pounds, K. A. 1994, MNRAS, 268, 405

Nandra, K., Pounds, K. A., \& Stewart, G. C. 1990, MNRAS, 242, 660

Nandra, K., Pounds, K. A., Stewart, G. C., Fabian, A. C., \& Rees, M. J. 1989, MNRAS, 236, 39P

Nandra, K., Pounds, K. A., Stewart, G. C., George, I. M., Hayashida, K., Makino, F., \& Ohashi, T. 1991, MNRAS, 248, 760

Netzer, H. 1976, MNRAS, 177, 473
Netzer, H. 1990a, in Variability of Active Galaxies, ed. W. J. Duschl, S. J. Wagner, \& M. Camenzind (Berlin: Springer), 107 . (Berlin: Springer), 57

1993, ApJ, 411, 594

Netzer, H., \& Maoz, D. 1990, ApJ, 365, L5

Osmer, P. S., Porter, A. C., \& Green, R. F. 1994, ApJ, 436, 678

Pan, H. C., Stewart, G. C., \& Pounds, K. A. 1990, MNRAS, 242, 177

Peterson, B. M. 1993, PASP, 105, 247

Pogge, R. W., \& Peterson, B. M. 1992, AJ, 103, 1084

Ptak, A., Yaqoob, T., Serlemitsos, P. J., Mushotzky, R., \& Otani, C. 1994, ApJ, 436, L31

Rees, M. J., Netzer, H., \& Ferland, G. J. 1989, ApJ, 347, 640

Reichert, G. A., Mushotzky, R. F., Petre, R., \& Holt, S. S. 1985, ApJ, 296, 69

Schulz, H. 1992, in Physics of Active Galactic Nuclei, ed. W. J. Duschl \& S. J. Wagner (Berlin: Springer), 235

Shields, J. C. 1994, in Reverberation Mapping of the Broad-Line Region of Active Galactic Nuclei, ed. P. M. Gondhalekar, K. Horne, \& B. M. Peterson (ASP Conf. Ser., 69), 293

Shields, J. C., \& Ferland, G. J. 1993, ApJ, 402, 425

Shull, J. M., \& Sachs, E. R. 1993, ApJ, 416, 536

Smith, L. J., \& Penston, M. V. 1988, MNRAS, 235, 551

Sparke, L. S. 1993, ApJ, 404, 570

Steidel, C. C., \& Sargent, W. L. W. 1991, ApJ, 382, 433

Stocke, J. T., Morris, S. L., Weymann, R. J., \& Foltz, C. B. 1992, ApJ, 396, 487

Turner, T. J., Nandra, K., George, I. M., Fabian, A. C., \& Pounds, K. A. 1993, ApJ, 419, 127

Tytler, D., \& Fan, X.-M. 1992, ApJS, 79, 1

Ulrich, M.-H. 1988, MNRAS, 230, 121

Voit, G. M., Shull, J. M., \& Begelman, M. C. 1987, ApJ, 316, 573

Walter, R., Fink, H. H., Orr, A., Courvoisier, T. J.-L., Makino, F., Otani, C., \& Wamsteker, W. 1994, A\&A, 285, 119

Wamsteker, W., \& Colina, L. 1986, ApJ, 311, 617

Weaver, K. A., et al. 1994, ApJ, 423, 621

Weymann, R. J. 1970, ApJ, 160, 31

Weymann, R. J., Morris, S. L., Foltz, C. B., \& Hewett, P. C. 1991, ApJ, 373, 23

White, R. J., \& Peterson, B. M. 1994, PASP, 106, 879

Wilkes, B. J. 1984, MNRAS, 207, 73

Williams, R. E., Strittmatter, P. A., Carswell, R. F., \& Craine, E. R. 1975, ApJ, 202, 296

Wu, C.-C., Boggess, A., \& Gull, T. R. 1983, ApJ, 266, 28

Yaqoob, T., Warwick, R. S., \& Pounds, K. A. 1989, MNRAS, 236, 153

Zamorani, G., et al. 1981, ApJ, 245, 357

Zheng, W. 1992, ApJ, 385, 127

Zheng, W., Kriss, G. A., Lee, G., \& Davidsen, A. F. 1992, BAAS, 24, 1209 